\title{
MRG-1 is required for genomic integrity in Caenorhabditis elegans germ cells
}

Jing $\mathrm{Xu}^{1,3}$, Xiaojuan Sun ${ }^{1,3}$, Yudong Jing ${ }^{1}$, Mo Wang ${ }^{2,3}$, Kai Liu ${ }^{1,3}$, Youli Jian ${ }^{1}$, Mei Yang ${ }^{1}$, Zhukuan Cheng ${ }^{2}$, Chonglin Yang ${ }^{1}$

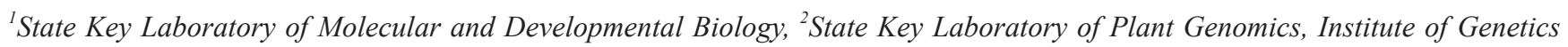
and Developmental Biology, Chinese Academy of Sciences, Beijing 100101, China; ${ }^{3}$ Graduate School, Chinese Academy of Sciences, Beijing 100309, China

During meiotic cell division, proper chromosome synapsis and accurate repair of DNA double strand breaks (DSBs) are required to maintain genomic integrity, loss of which leads to apoptosis or meiotic defects. The mechanisms underlying meiotic chromosome synapsis, DSB repair and apoptosis are not fully understood. Here, we report that the chromodomain-containing protein MRG-1 is an important factor for genomic integrity in meiosis in Caenorhabditis elegans. Loss of $\mathrm{mrg}-1$ function resulted in a significant increase in germ cell apoptosis that was partially inhibited by mutations affecting DNA damage checkpoint genes. Consistently, mrg-1 mutant germ lines exhibited SPO-11-generated DSBs and elevated exogenous DNA damage-induced chromosome fragmentation at diakinesis. In addition, the excessive apoptosis in $\mathbf{m r g}$ - $\mathbf{1}$ mutants was partially suppressed by loss of the synapsis checkpoint gene pch-2, and a significant number of meiotic nuclei accumulated at the leptotene/zygotene stages with an elevated level of H3K9me2 on the chromatin, which was similarly observed in mutants deficient in the synaptonemal complex, suggesting that the proper progression of chromosome synapsis is likely impaired in the absence of $m r g-1$. Altogether, these findings suggest that MRG-1 is critical for genomic integrity by promoting meiotic DSB repair and synapsis progression in meiosis.

Keywords: apoptosis; DSB repair; synapsis; meiosis; genomic integrity; C. elegans

Cell Research (2012) 22:886-902. doi:10.1038/cr.2012.2; published online 3 January 2012

\section{Introduction}

Genomic integrity is a prerequisite for the survival, development and propagation of eukaryotic organisms. The genome is, however, frequently attacked by either endogenous or exogenous DNA-damaging agents that generate a wide variety of DNA lesions. To maintain the integrity of their genomes, eukaryotic organisms have developed multiple evolutionarily conserved strategies to repair the lesions caused by DNA-damaging insults [1]. When DNA lesions are too severe to repair, cells are destined to undergo apoptosis so as to avoid their deleterious propagation. Defective DNA repair or abnormal apoptosis leads to genomic instability, which is a hallmark of cancer [2]. On the other hand, the maintenance

Correspondence: Chonglin Yang

E-mail: clyang@genetics.ac.cn

Received 2 May 2011; revised 19 August 2011; accepted 18 October 2011; published online 3 January 2012 of genomic integrity is required for faithful transmission of genetic information from parents to progeny through meiosis, a specialized cell division program in which DNA replicates once followed by two successive cell divisions. During the prophase of meiosis I, chromosomes undergo a series of programmed events, including sister chromatid cohesion, pairing of homologous chromosomes, synapsis, and homologous recombination [3]. Defects in any of these steps may result in aberrant chromosome segregation, leading to sterility, $\mathrm{X}$ chromosome non-disjunction, aneuploidy, or embryonic lethality $[3,4]$.

The regulatory mechanisms underlying the maintenance of genomic integrity in the process of meiosis are evolutionarily conserved. In the nematode $C$. elegans, genetic and cell biological studies have revealed key components required for distinct chromosomal events in the prophase of meiosis I [3-5]. Following DNA replication, chromosome condensation begins and homologous chromosomes pair and align longitudinally. The meiosis-specific protein REC-8 and some $\mathrm{COH}$ (ohesion protein homologs) 
paralogs are essential for sister chromatid cohesion, while the zinc-finger proteins HIM-8, ZIM-1, -2, and -3 promote chromosome pairing and synapsis $[3,5,6]$. The proteinaceous synaptonemal complex (SC), which consists of the lateral elements HTP-1, $-2,-3$, and HIM-3 and the central elements SYP- $1,-2,-3$ and -4 , forms a zipperlike structure to hold homologous chromosomes together along their entire lengths. Loss-of-function mutations (lf) in SC components lead to synaptic failure, which manifests as the presence of up to 12 univalents in diakinesis oocytes, compared with 6 bivalents representing six wellpaired homologous chromosomes in wild-type oocytes $[3,5,7,8]$. Concomitant with the morphological reorganization of chromosomes, DNA molecules also undergo programmed double strand breaks (DSBs) and their repair results in the formation of crossovers between homologous chromosomes. DSBs are normally induced by an evolutionarily conserved topoisomerase-like endonuclease, SPO-11, loss of which leads to failure in crossover. Consequently spo- 11 mutants show 12 univalents in oocytes in C. elegans [9]. In contrast, loss of rad-51, which encodes a RecA-related protein required for DSB repair, causes severe defects in chromosome morphology [10]. Likewise, mutants for other genes required for DSB repair display phenotypes of altered chromosome morphology, aneuploidy, sensitivity to DNA damage, and developmental retardation or even embryonic lethality, all of which are indicative of chromosomal instability [4]. In addition, defective DSB repair in C. elegans germ cells manifests as the high-incidence-of-males (Him) phenotype owing to $\mathrm{X}$ chromosome non-disjunction. Notably, while meiotic DSBs are required for SC formation and thus synapsis in yeast, plants and mammals, DSB formation and synapsis are largely independent of one another in $C$. elegans $[9,11,12]$. This makes $C$. elegans an excellent model organism for identifying novel factors and further dissecting their functions in either synapsis or meiotic DSB repair, or both.

In the C. elegans hermaphrodite germ line, either synaptic failure or defective DSB repair leads to an elevation in apoptosis, indicating that apoptosis is an essential cellular event for maintenance of genomic integrity [13, 14]. Nevertheless, synaptic failure and defective DSB repair appear to trigger the cell death program by distinct signaling pathways. DNA lesions resulting from either defective meiotic DSB repair or exogenous genotoxic stress are sensed by checkpoint genes hus-1, mrt-2/radl and/or $c l k-2 / \mathrm{rad} 5$, which transduce death signals to the effector protein CEP-1, the C. elegans homolog of mammalian p53 [14]. As a transcription factor, CEP-1 upregulates the expression of the cell death initiator EGL-1, a BH3-only protein homologous to the mammalian p53 targets PUMA and Noxa, to induce apoptosis through an evolutionarily conserved cell death machinery [15-17]. In comparison, synaptic failure originating from defects in the pairing center or mutations in the SC triggers apoptosis through the synapsis checkpoint $\mathrm{PCH}-2$, a C. elegans homolog of the yeast AAA-adenosine triphosphatase (AAA-ATPase) Pch2 [13], though it remains unknown how $\mathrm{PCH}-2$ mediates death signals to the death initiator. The requirement of distinct death signaling pathways by synaptic failure or defective DSB repair further provides a basis for analyzing the function of genes involved in genomic integrity.

Here, we report the identification of MRG-1 as an important factor for genomic integrity during the meiotic process in C. elegans. Worm MRG-1 is a homolog of the mammalian mortality factor-related protein MRG15, which is conserved in yeast, plants, and Drosophila [18, 19]. In mammals, MRG15 is a chromodomain-containing protein that potentially regulates both transcriptional silencing and activation by associating with both histone deacetylases and histone acetyltransferase (HAT) complexes [20]. Deletion of Mrg 15 in mouse causes embryonic lethality, improper organogenesis and proliferation, and defective DNA damage repair [21]. Nevertheless, it remains elusive how MRG15 is involved in these processes at the molecular level. In C. elegans, $\mathrm{mrg}^{-1}$ was found to promote embryo survival, and maternal MRG1 is required for proliferation and immortality of the primordial germ cells (PGC) [18]. MRG-1 is essential for maintaining repression of transgenes in the maternal germ line by associating with chromatin in autosomes but not the X chromosome [18]. Remarkably, repression of several X-linked genes is dependent on autosomeassociated MRG-1 in conjunction with the "maternaleffect sterile" protein MES-4, a methyltransferase that modifies H3K36 [22]. These findings suggest that MRG1 acts at the chromatin level to regulate germline potential and immortality. In the present study, we found that loss of $m r g-1$ function leads to excessive apoptosis in the C. elegans germ line, which requires both DNA damage checkpoint genes, such as $c l k-2 / \mathrm{rad} 5$, and the chromosome synapsis checkpoint gene $p c h-2$. Consistent with this, we found that meiosis is defective in $m r g-1$ mutants, probably due to defects in DSB repair and synapsis. These results suggest that MRG-1 plays an important role in maintenance of genomic integrity during meiosis.

\section{Results}

Loss of mrg-1 function causes increased germ cell apoptosis

To identify novel factors involved in germ cell apopto- 
sis in C. elegans, we performed a genome-wide RNA interference (RNAi) screen for genes that when inactivated can reduce or enhance the number of germ cell corpses in vps-18(tm1125) mutants, which accumulate germ cell corpses as a result of defective corpse degradation [23]. We found that RNAi inactivation of $m r g-1$, a germline mortality-related gene $[18,19]$, enhanced the number of germ cell corpses in vps-18(tm1125) mutants in an agedependent manner (Figure 1A). In wild-type worms or worms carrying an integrated array (smIs34) expressing the phagocytic receptor CED-1 fused with green fluorescence protein (CED-1::GFP) [24], mrg- 1 RNAi also led to an obvious increase in germ cell corpses as viewed by either formation of disc-like structures under differential interference (DIC) optics or formation of CED-1::GFP rings at all adult ages examined (Figure $1 \mathrm{~B}$ and $1 \mathrm{C}$ ). Similarly, mrg-1 deletion mutants, ok1262, qa6200, and tm 1227 [18], showed a significantly higher level of apoptosis than wild-type worms at most adult ages examined (Figure 1B and 1D). Notably, the number of germ cell corpses in $m r g-1(R N A i)$ worms appeared higher than in mrg- 1 deletion mutants (Figure 1C and 1D, and see Figure $1 \mathrm{E}$ and $1 \mathrm{~F}$ ), probably because $m r g-1$ RNAi had a less severe effect on the proliferation of germ cells than $\mathrm{mrg}$ 1 deletion such that $m r g-1(R N A i)$ worms possessed more germ cells competent for apoptosis than the deletion mutants. For example, $\operatorname{mrg}-1(R N A i)$ worms contained an average of $142.3 \pm 8.1$ (SEM, standard error of the mean; $n$ =14) mitotic cells per gonad arm, compared with 197.8 $\pm 7.1(n=14)$ mitotic cells per gonad arm in wild type, whereas mrg-1(qa6200) hermaphrodites only had an average of $101.3 \pm 2.8(n=13)$ mitotic cells per gonad arm. These results indicate that loss of $m r g-1$ function induced apoptosis in the C. elegans germ line.

C. elegans MRG-1 is homologous to yeast Eaf3/ Alp13, Drosophila dMrg15, and mammalian MRG15, all of which are implicated in repair of DNA DSBs caused by exogenous DNA-damaging agents [19, 25-28]. During meiosis of $C$. elegans hermaphrodite, failure to repair programmed DSBs or artificially induced breaks leads to apoptotic cell death. To test whether loss of $\mathrm{mrg}-1$ function confers sensitivity to DNA damage, we exposed $m r g-1(l f)$ worms to DNA-damaging agents and examined germ cell apoptosis. Both $\gamma$-irradiation, which mainly induces DSBs, and $N$-ethyl- $N$-nitrosourea (ENU), which causes a wide spectrum of DNA lesions, induced a significant increase in germ cell corpses in $m r g-1(l f)$ worms in a dose- or concentration-dependent manner compared with wild-type worms (Figure $1 \mathrm{E}$ and $1 \mathrm{~F}$ ). This suggests that loss of $m r g-1$ function likely causes defective DNA repair in germ cells, which in turn results in excessive apoptosis following exposure to DNA-damaging insults.
The increase in germ cell apoptosis in the absence of mrg-1 partially requires DNA damage checkpoint signaling

To understand the mechanisms underlying the increased apoptosis in $m r g-1$ mutants, we investigated whether the DNA damage signaling pathway plays a role. In C. elegans, germ cell apoptosis resulting from irreparable DNA lesions is dependent on DNA damage checkpoint genes that function in two parallel groups to mediate death signals to the downstream effector CEP-1/ $\mathrm{p} 53$, with hus-1, mrt-2/radl in one group and clk-2/rad5 in the other. Mutations in these genes suppress germ cell apoptosis in response to DNA damage [15, 29-32]. Since $m r g-1(R N A i)$ worms showed excessive germ cell apoptosis similarly to other $m r g-1$ mutants (Figure 1), we performed $m r g-1$ RNAi in mutants of the DNA damage checkpoint genes hus-1, mrt-2, and clk-2. Interestingly, the numbers of germ cell corpses in $m r g-1$ RNAi-treated hus-1(op244) and mrt-2(e2663) mutants were not obviously decreased compared with that in $m r g-1$ RNAitreated wild type at the adult ages examined, whereas $m r g-1$ RNAi-induced cell corpses were substantially reduced, though not totally blocked, in clk-2(mn159) germ lines (Figure 2A), suggesting that the DNA damage checkpoint gene $c l k-2 / \mathrm{rad} 5$ is partially required for germ cell apoptosis in the absence of $m r g-1$. Similarly, germ cell corpses were significantly reduced but not completely eliminated in $m r g-1$ RNAi-treated cep- $1(g k 138)$ deletion mutants and double mutants of cep-1 ( $g k 138)$ with individual $m r g-1$ alleles (Figure $2 \mathrm{~A}$ and $2 \mathrm{~B}$ ). Based on these observations, we reasoned that the increase in germ cell apoptosis in $\mathrm{mrg}-1(\mathrm{lf})$ worms were partially due to endogenous DNA lesions. In agreement with this, we found that the number of germ cell corpses in $m r g-1$ RNAi-treated spo-11(ok79) mutants, in which no DSBs were generated (see below) was only reduced to a similar level to that in cep-1 (gk138); $m r g-1(R N A i)$ worms (Figure 2A). Furthermore, in double mutants of cep-1 (gk138) with spo-11(ok79), $m r g-1$ RNAi still caused a significant increase in germ cell apoptosis compared with control RNAi treatment (Figure 2A). Altogether, these data suggest that germ cell apoptosis in the absence of $m r g-1$ is partially attributable to defective DSB repair that signals through the DNA damage checkpoint gene $c l k-2 / r a d 5$ to CEP-1/p53.

\section{Loss of mrg-1 function affects DSB repair}

As the increased germ cell apoptosis in $\mathrm{mrg}-1$ mutants is partially dependent on the DNA damage signaling pathway, we next investigated whether germline nuclei of $m r g-1$ mutants indeed contained excessive unrepaired DSBs. To this end, we analyzed RAD-51 foci in consecu- 
tive germline regions as described previously [33]. RAD51 is a RecA recombinase required for strand invasion/ exchange during HR-mediated DSB repair by binding to single-stranded DNA with a $3^{\prime}$ overhang and forming a nucleoprotein filament [34]. In wild-type germ lines, RAD-51 foci mostly appeared at the early pachytene

A

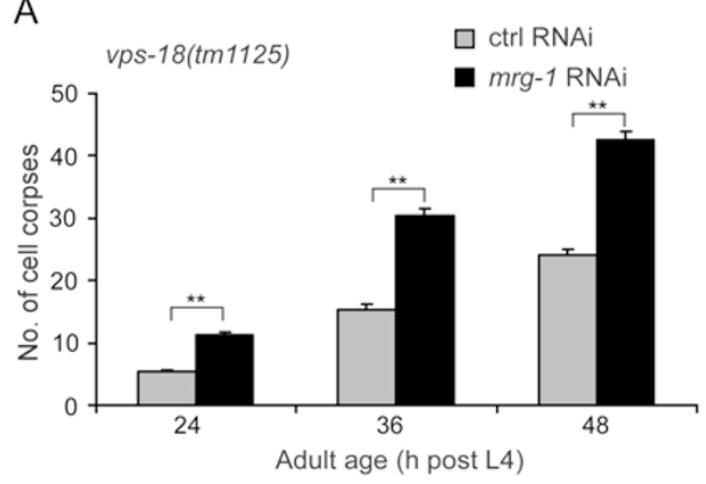

C

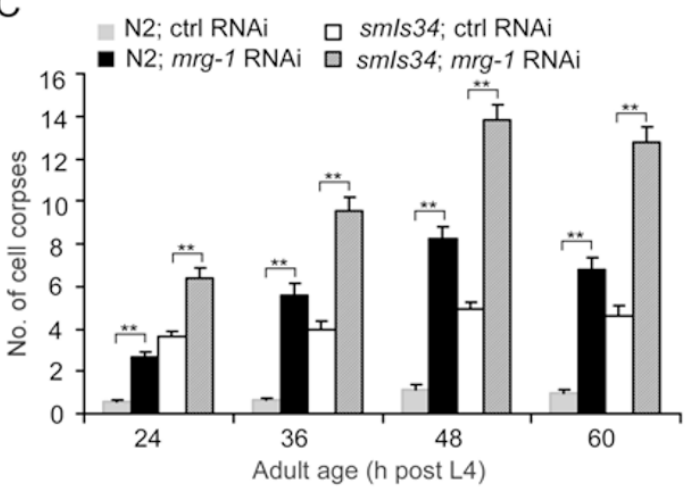

E

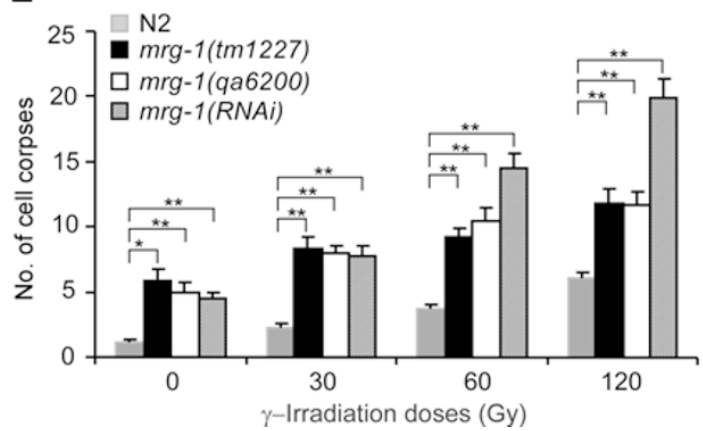

B

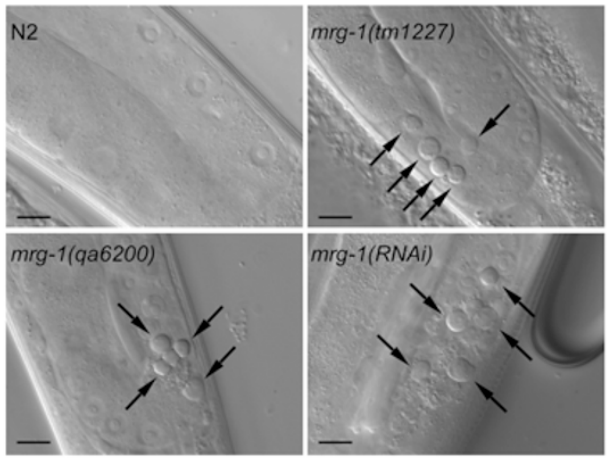

D

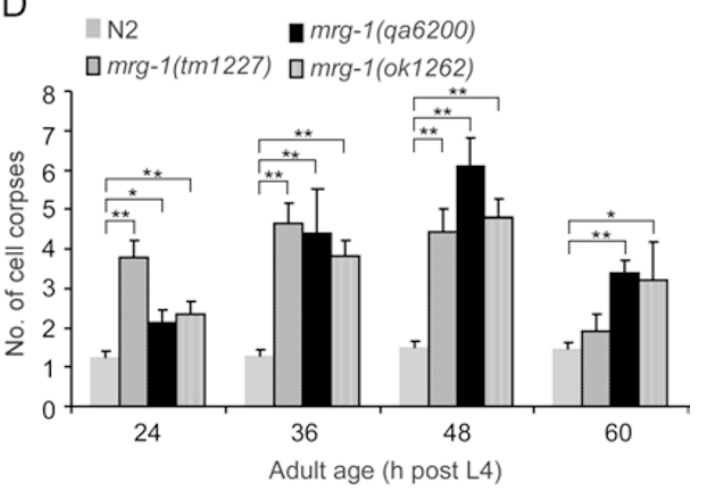

$\mathrm{F}$

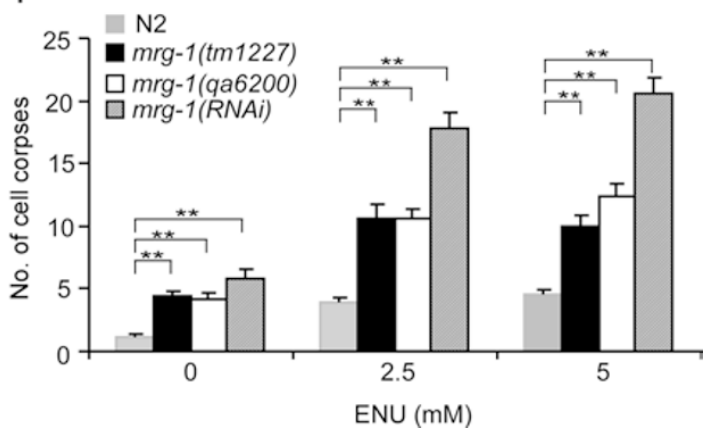

Figure 1 Loss of mrg-1 function induces germ cell apoptosis. (A) mrg-1 RNAi enhances the number of germ cell corpses in vps-18(tm1125) mutants. (B) Representative DIC images of germ cell corpses in wild-type (N2), mrg-1(qa6200), mrg1(tm1227) and mrg-1(RNAi) worms. Arrows indicate cell corpses. Bars, $10 \mu \mathrm{m}$. (C) Time course analyses of germ cell corpses in wild-type and sm/s34 worms treated with $m r g-1$ RNAi. In wild type, cell corpses were viewed by formation of disclike structures under DIC optics. In sm/s34 worms, cell corpses were viewed by formation of CED-1::GFP rings. (D) $m r g-1$ deletion mutants show increased germ cell apoptosis. (E, F) Quantification of germ cell apoptosis induced by $\gamma$-irradiation (E) and ENU (F) in mrg-1 mutants. Cell corpses were scored and analyzed as described in Materials and Methods. Error bars represent standard error of the mean (SEM). Comparisons were performed using unpaired $t$-test. ${ }^{*} P<0.05,{ }^{* *} P<0.001$. 

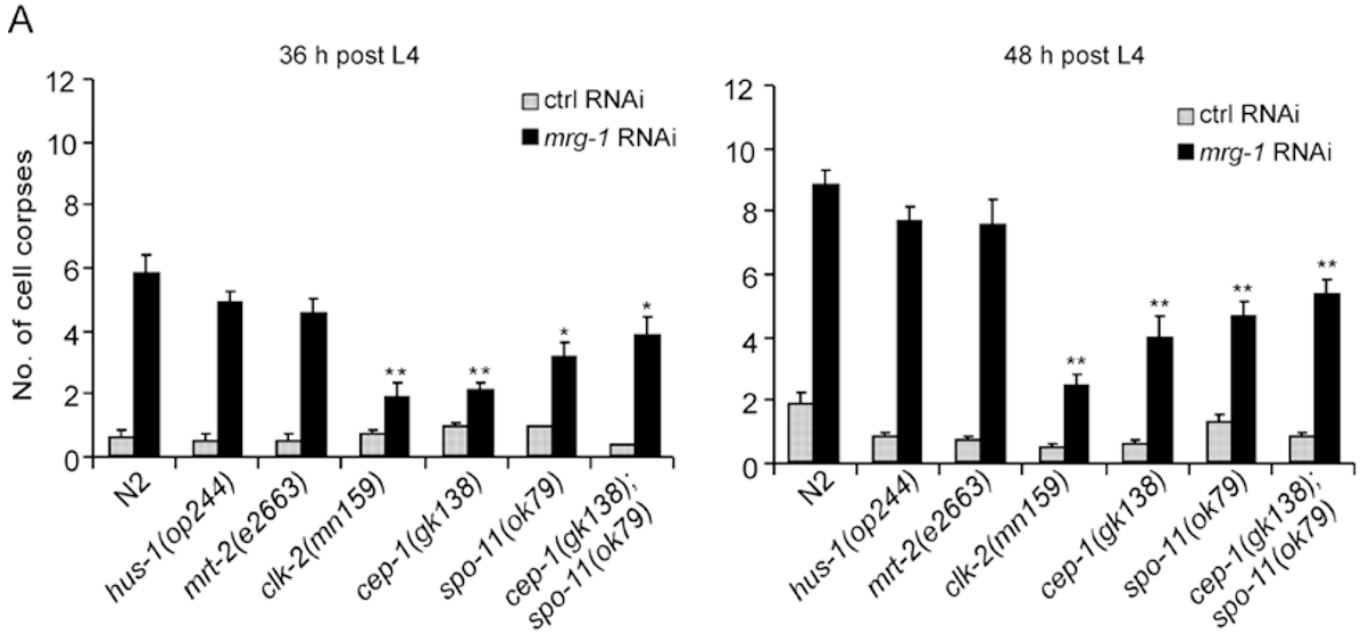

B
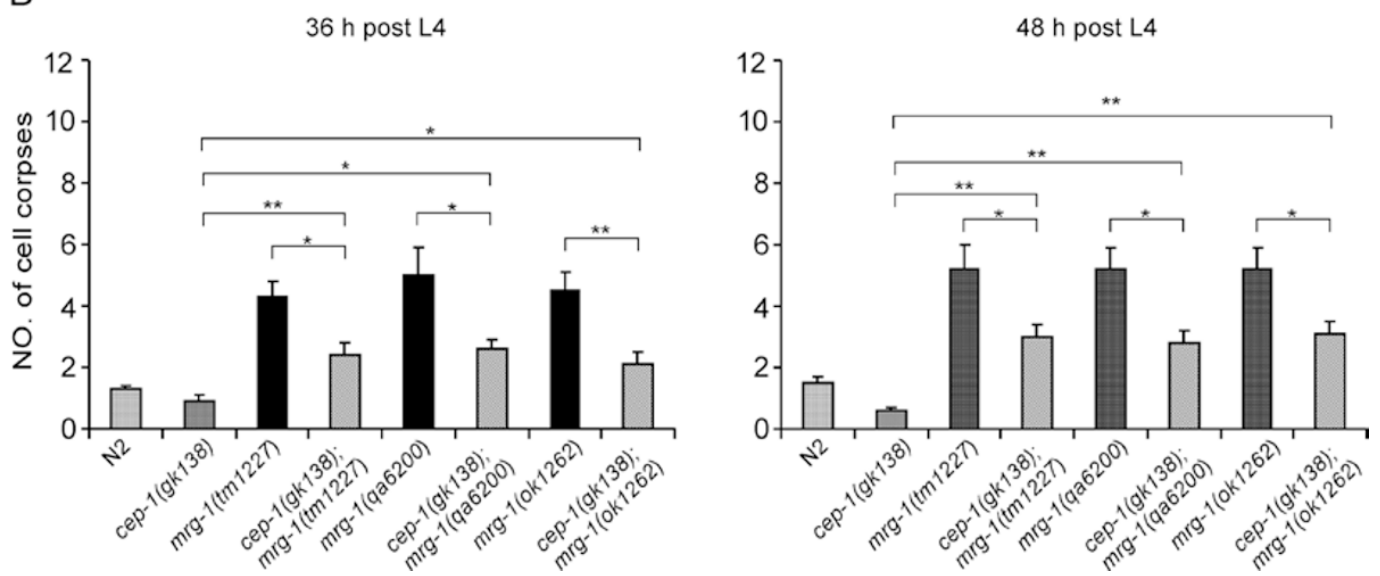

Figure 2 Mutations affecting DNA damage checkpoint genes partially suppress germ cell apoptosis induced by $m r g-1$ RNAi. (A) Germ cell corpses in mrg-1 RNAi-treated mutants including hus-1(op244), mrt-2(e2663), clk-2(mn159), cep-1(gk138), spo11(ok79) and cep-1(gk138);spo-11(ok79) were quantified and analyzed at the adult ages of $36 \mathrm{~h}$ (left) and $48 \mathrm{~h}$ (right) post L4 stage, respectively. Comparisons were made between N2 and other strains treated with mrg-1 RNAi using unpaired $t$-test. ${ }^{*} P<0.05,{ }^{* *} P<0.001$. (B) Quantification of germ cell corpses in N2, cep-1(gk138) and double mutants of cep-1(gk138) with $m r g-1(t m 1227), m r g-1(q a 6200)$ and $m r g-1$ (ok1262) at 36 and 48 h post L4 stage. ${ }^{*} P<0.05,{ }^{* *} P<0.001$.

early pachytene stage, which peaked in the mid pachytene stage and persisted until the late pachytene stage (Figure $3 \mathrm{~A}$ and $3 \mathrm{~B})$. In contrast, RAD-51 foci were barely induced in $m r g-1$ RNAi-treated spo-11(ok79) null mutants, indicating that the accumulation of DSBs in $m r g-1 \mathrm{mu}-$ tants is dependent on SPO-11 (Figure 3A and 3B). Thus loss of $m r g-1$ function leads to accumulation of DSBs that likely result from a failure of timely processing.

To further determine the role of MRG-1 in DSB repair in C. elegans, we treated $m r g-1(l f)$ worms at L4 stage with $\gamma$-irradiation and examined the appearance of fragmented chromosomes in oocytes using a procedure described previously [35]. In wild-type worms, $\gamma$-irradiation of 70 and 90 Gy induced chromosome fragmentation in
$8.8 \%$ and $27.1 \%$ oocytes, respectively (Figure $4 \mathrm{~A}$ and 4B). In $m r g-1(l f)$ and $m r g-1(R N A i)$ worms, the proportion of oocytes with fragmented chromosomes following $\gamma$-irradiation were greatly increased, ranging from $42.3 \%$ $64.0 \%$ and $70.8 \%-87.7 \%$ with irradiation of 70 and 90 Gy, respectively (Figure 4A and 4B). In addition, an irradiation of $m r g-1$ (qa6200) L1 worms with a lower dose of $\gamma$-ray (50 Gy) led to only about $40 \%$ worms growing to sterile adults, compared with around $93 \%$ of N2 L1 worms growing to fertile adults with the same treatment (data not shown). Altogether, these findings suggest that mrg- 1 plays an important role in DSB repair to maintain genomic integrity. 
mrg-1 mutants exhibit meiotic defects

Our finding that $m r g-1$ is important for DSB repair in meiosis prompted us to further examine whether $m r g-1$ mutants exhibited meiotic phenotypes such as high in-
A

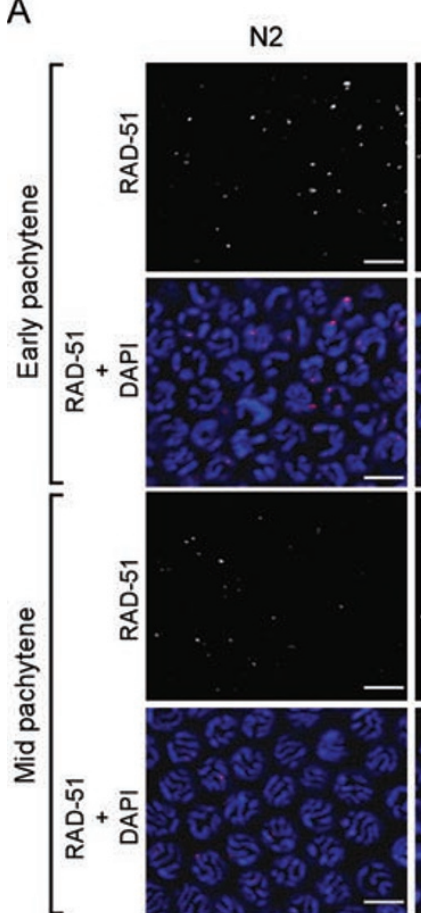

[

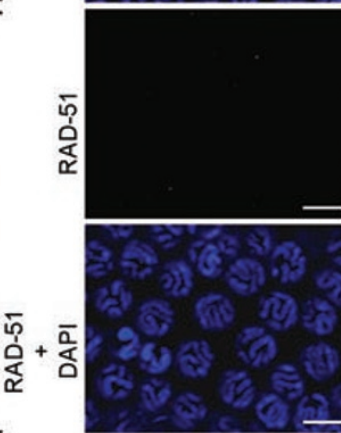

B
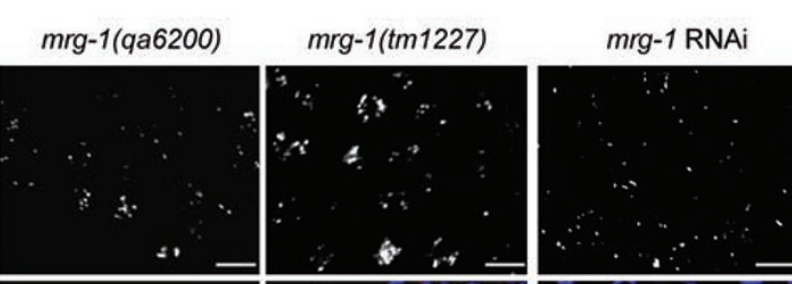

spo-11(ok79);

mrg-1 RNAi
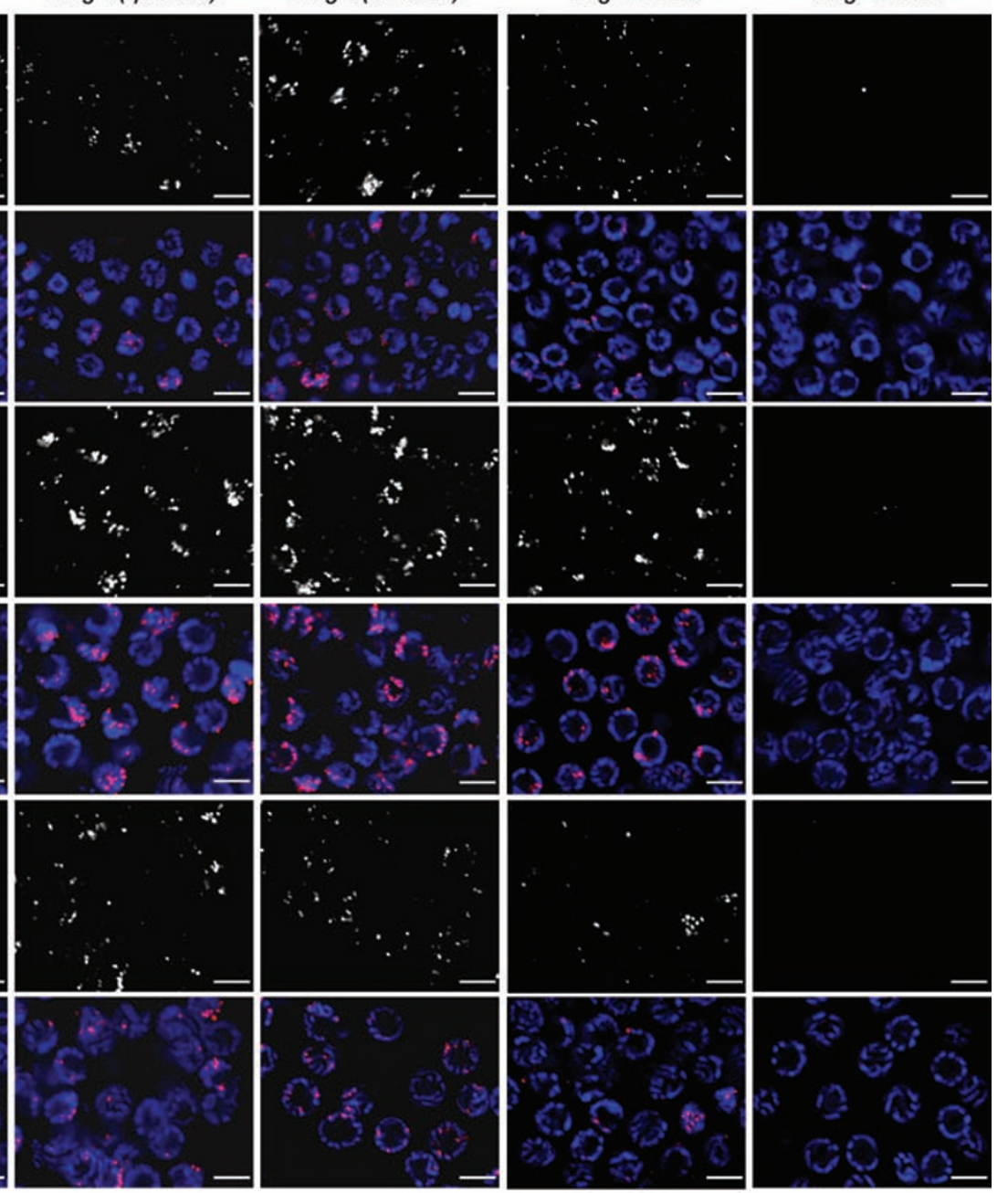

Mid pachytene

Late pachytene
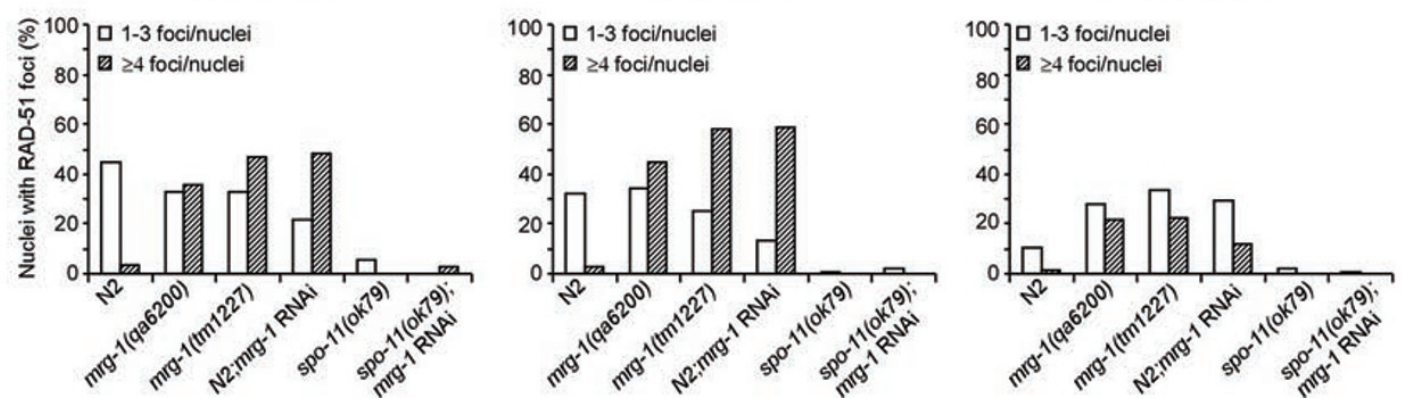

Figure 3 mrg-1 mutants accumulate RAD-51 foci in the germ line. (A) Representative images of RAD-51 foci and merged images of RAD-51 foci and DAPI staining are shown for early, mid and late pachytene nuclei of N2, mrg-1(qa6200), mrg1(tm1227), $m r g-1(R N A i)$, and spo-11(ok79);mrg-1(RNAi) worms. Bars, $5 \mu \mathrm{m}$. (B) Quantification of RAD-51 foci in early, mid and late pachytene stages of germ lines in worms as indicated. For each region, at least 20 nuclei per gonad arm were analyzed for a total of $\geq 3$ gonads. 
cidence of males (Him), which reflects the frequency of $\mathrm{X}$ chromosome non-disjunction, and increased number of 4',6-diamidino-phenylindole (DAPI)-stained bodies at diakinesis, which reflects defective crossover formation and/or DNA repair [4]. It was shown previously that $m r g$ 1 is required for proliferation and immortality of PGC and the brood sizes of $m r g-1(l f)$ and $m r g-1(R N A i)$ worms were greatly reduced, with a substantial proportion of embryos inviable [18] (Figure 5A). Among the surviving progeny, however, around 3.1\%-9.5\% were males, which is significantly higher than in wild type (Figure 5A). This indicates that loss of $\mathrm{mrg}-1$ leads to $\mathrm{X}$ chromosome nondisjunction. Moreover, approximately $8 \%-17 \%$ oocyte nuclei in $\mathrm{mrg}-1(\mathrm{lf})$ worms contained more than six DAPI-stained bodies, varying from 7 to 12 (Figure 5B and $5 \mathrm{C}$ ), which probably resulted from defects in crossover formation.

To determine the effect of apoptosis on meiosis in $m r g-1(l f)$ animals, we analyzed the meiotic phenotype in double mutants of $\mathrm{mrg}-1$ (qa6200) with ced-3(n717), a strong loss-of-function allele of the ced-3 gene that is required for cell death in C. elegans. No germ cell death

A
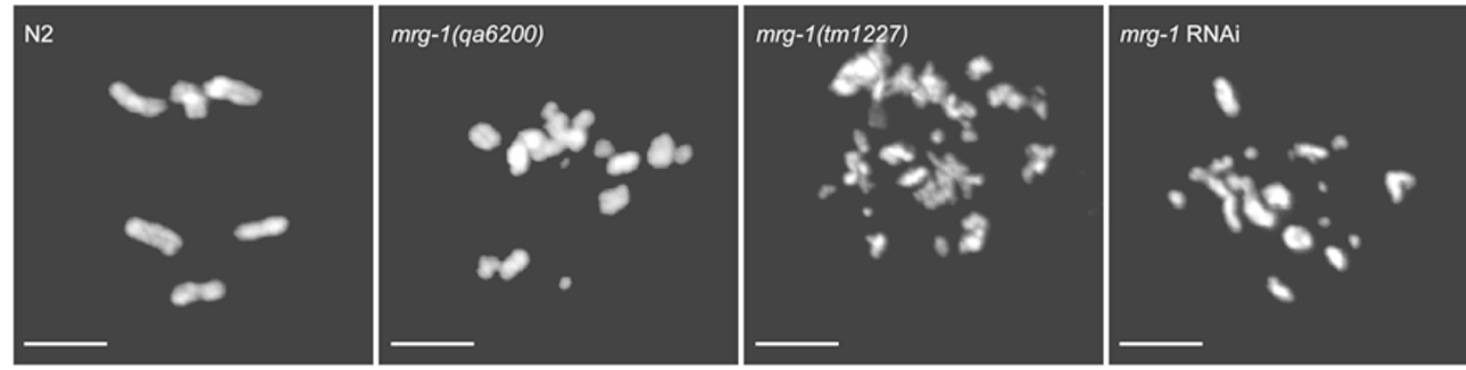

B

\begin{tabular}{lccccc}
\hline & \multicolumn{2}{c}{$70 \mathrm{~Gy}$} & & \multicolumn{2}{c}{$90 \mathrm{~Gy}$} \\
\cline { 2 - 3 } \cline { 5 - 6 } Strain & $\begin{array}{c}\text { \% of nuclei with> } 6 \\
\text { DAPI-stained bodies }\end{array}$ & $\begin{array}{c}\text { \# of } \\
\text { nuclei scored }\end{array}$ & & $\begin{array}{c}\text { \% of nuclei with }>6 \\
\text { DAPI-stained bodies }\end{array}$ & $\begin{array}{c}\text { \# of } \\
\text { nuclei scored }\end{array}$ \\
\hline N2 & 8.8 & 137 & 64 & 27.1 & 203 \\
$m r g-1$ (qa6200) & 64.0 & 75 & 87.7 & 90 \\
$m r g-1$ (tm1227) & 51.6 & 52 & 86.7 & 114 \\
$m r g-1$ RNAi & 42.3 & & 70.8 & 72 \\
\hline
\end{tabular}

C $\%$ of nuclei with $>6$ DAPI-stained bodies (total number scored)

\begin{tabular}{llllll}
\hline \multirow{2}{*}{ Strain } & \multicolumn{2}{c}{70 Gy } & & \multicolumn{2}{c}{$90 \mathrm{~Gy}$} \\
\cline { 2 - 3 } \cline { 5 - 6 } N2 & Ctrl RNAi & met-2 RNAi & & Ctrl RNAi & met-2 RNAi \\
\hline mrg-1(qa6200) & $9.3(86)$ & $12.9(139)$ & & $17.4(69)$ & $20.2(119)$ \\
$m r g-1$ (tm1227) & $57.4(108)$ & $54.8(62)$ & & $73.1(108)$ & $74.7(146)$ \\
\hline
\end{tabular}

Figure $4 \gamma$-irradiation induced chromosome fragmentation in N2 and mrg-1(If) worms. (A) Representative images of chromosomes in oocytes of N2 and $\mathrm{mrg}$-1(If) worms treated with $\gamma$-irradiation of $70 \mathrm{~Gy}$. Bars, $5 \mu \mathrm{m}$. (B) Quantification of chromosome fragmentation in oocyte nuclei with $\gamma$-irradiation of 70 and $90 \mathrm{~Gy}$, respectively. (C) Quantification of chromosome fragmentation in oocyte nuclei of met-2 RNAi-treated worms as indicated after exposure to $\gamma$-irradiation of 70 and 90 Gy, respectively. The percentages of nuclei with $>6$ DAPI-stained bodies are shown and the total numbers of nuclei scored are shown in parentheses. 
was observed in $\mathrm{mrg}$-1(qa6200); ced-3(n717) double mutants (data not shown), but their brood size and survival of embryos were strongly decreased compared with that of mrg-1 (qa6200) and ced-3(n717) single mutants (Figure $5 \mathrm{~A})$. Notably, up to $36.7 \%$ of the surviving progeny of $\mathrm{mrg}-1$ (qa6200); ced-3(n717) double mutants were males, which is more than three times as many as in $\mathrm{mrg}$ 1(qa6200) single mutants (Figure 5A). These findings suggest that apoptosis is required to eliminate cells that harbor meiotic defects resulting from loss of $m r g-1$ function.

Apoptosis caused by loss of mrg-1 function involves the synapsis checkpoint

Our results indicate that $m r g-1$ is important for meiotic DSB repair. However, the elevated apoptosis in $m r g-1(l f)$ germ lines is only partially dependent on the DNA damage checkpoint signaling pathway, suggesting that other checkpoint signals are also required. In the $C$. elegans germ line, defective chromosome synapsis in the prophase of meiosis I was found to trigger germ cell apoptosis [13]. To test whether synapsis checkpoint signaling participates in germ cell apoptosis in $m r g-1(l f)$ worms, we performed $m r g-1$ RNAi in pch-2(tm 1458) deletion mutants that affect the synapsis checkpoint gene $p c h$ 2. As shown in Figure 6A, mrg-1 RNAi-induced germ cell apoptosis was partially but significantly reduced in pch-2(tm1458) mutants at all adult ages examined, suggesting that the synapsis checkpoint is involved in the increase of germ cell apoptosis in the absence of $\mathrm{mrg}$ 1. Next, we tested whether inactivation of both DNA damage and synapsis checkpoint pathways could abolish mrg-1 RNAi-induced germ cell apoptosis. In double mutants of pch-2(tm1458) with spo-11(ok79), mrg-1 RNAi did not cause an obvious increase in germ cell apoptosis compared with control RNAi (Figure 6A). Similarly, no increase in germ cell apoptosis was observed in cep1(gk138);pch-2(tm 1458) double mutants following mrg- 1 RNAi treatment (Figure 6A). In addition, $m r g-$ 1 RNAi failed to induce obvious apoptosis in the egl1(n1084n3082) strong loss-of-function mutants affecting the cell death initiator EGL-1 (Figure 6A). These results indicate that loss of $m r g-1$ function activated both synapsis and DNA damage checkpoint signaling pathways to trigger apoptosis (Figure 6B).

mrg-1 mutant germ lines are defective in progression of meiotic prophase

We next investigated whether chromosome synapsis is affected in $m r g-1$ mutants. In wild-type germ lines syncytial nuclei were spatially arranged according to the temporal progression of the prophase of meiosis I [3]. Nuclei undergoing mitosis and premeiotic replication were in the distal most end of the gonad, which was followed by a transition zone where nuclei entered leptotene/zygotene

\begin{tabular}{llll} 
A & & \\
\hline Strain & Brood size $(\mathrm{n})$ & $\begin{array}{c}\text { Hatching } \\
(\%)\end{array}$ & $\begin{array}{l}\text { Male } \\
(\%)\end{array}$ \\
\hline $\mathrm{N} 2$ & $259.4 \pm 7.8(16)$ & $99.4 \pm 1.2$ & 0.1 \\
mrg-1(RNAi) & $96.8 \pm 6.2(12)$ & $47.8 \pm 4.5$ & 3.1 \\
mrg-1(tm1227) & $59.8 \pm 3.7(60)$ & $56.3 \pm 3.6$ & 3.9 \\
mrg-1(qa6200) & $52.2 \pm 7.0(52)$ & $30.6 \pm 6.4$ & 9.5 \\
ced-3(n717) & $221.7 \pm 10.3(12)$ & $89.7 \pm 0.0$ & 0.9 \\
mrg-1(qa6200); & $1.7 \pm 0.4(82)$ & $12.0 \pm 2.1$ & 36.7 \\
ced-3(n717) & & & \\
\hline
\end{tabular}
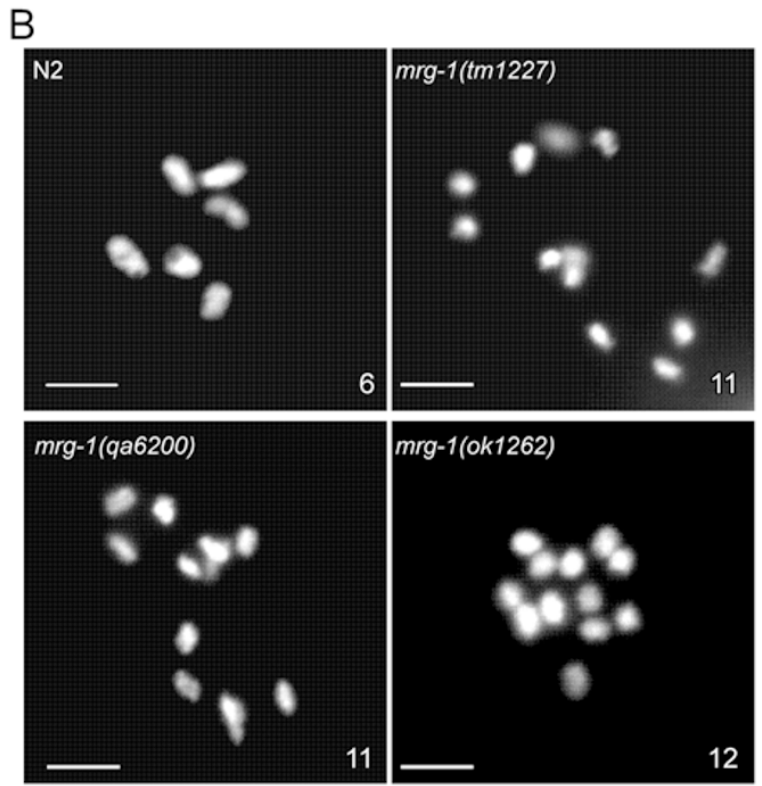

C

\begin{tabular}{lcc}
\hline Strain & $\begin{array}{c}\text { Nuclei with >6 DAPI- } \\
\text { stained bodies (\%) }\end{array}$ & $\begin{array}{c}\text { No. of diakinesis } \\
\text { nuclei scored }\end{array}$ \\
\hline N2 & 0 & 234 \\
$m r g-1(t m 1227)$ & 8.6 & 273 \\
$m r g-1(q a 6200)$ & 16.9 & 332 \\
$m r g-1$ (ok1262) & 7.7 & 362 \\
mrg-1(RNAi) & 7.5 & 148 \\
\hline
\end{tabular}

Figure 5 mrg-1 mutants are defective in meiosis. (A) Quantification of brood sizes, hatching and male percentages of N2, $\mathrm{mrg}$ 1(RNAi), mrg-1(qa6200), mrg-1(tm1227), ced-3(n717) and $m r g-$ 1(qa6200);ced-3(n717) worms. (B) mrg-1 mutant oocytes show $>6$ DAPI-stained bodies. Bars, $5 \mu \mathrm{m}$. (C) Quantification of $\mathrm{mrg}$ 1 mutant oocytes with $>6$ DAPI-stained bodies. 
A
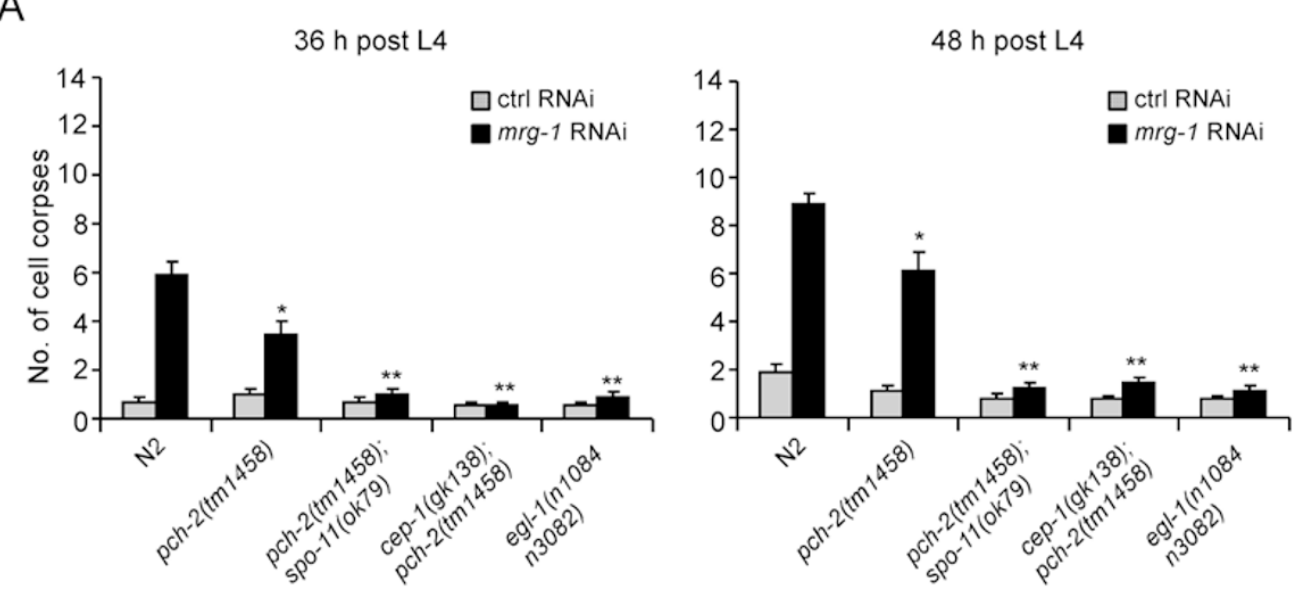

B

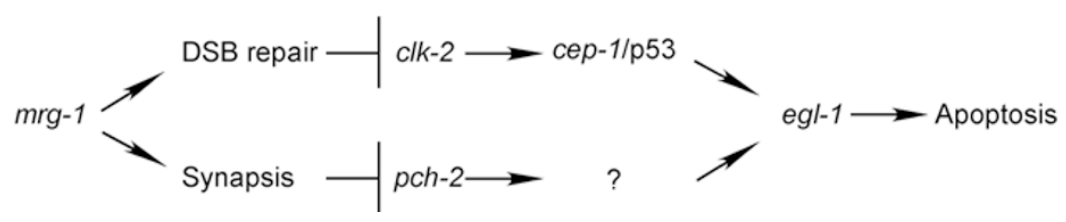

Figure 6 The synapsis checkpoint gene pch-2 and DNA damage checkpoint genes are required for increased apoptosis in mrg-1(RNAi) worms. (A) Quantification of germ cell apoptosis in mrg-1 RNAi-treated N2, pch-2(tm1458), pch-2(tm1458);spo11(ok79), cep-1(gk138);pch-2(tm1458), and egl-1(n1084n3082) worms. Germ cell corpses in RNAi-treated worms at adult ages of $36 \mathrm{~h}$ (left) and $48 \mathrm{~h}$ (right) post L4 stage were scored and analyzed as described in Materials and Methods. Comparisons were made between N2 and other mutants treated with $m r g-1$ RNAi. ${ }^{*} P<0.05$, ${ }^{* *} P<0.001$. (B) A schematic summary of the signaling pathways leading to apoptosis in the absence of $m r g-1$.

and the chromatin polarized, adopting a crescent-like morphology. In pachytene, the chromatin redispersed and chromosomes synapsed, which could be visualized as parallel tracks when stained with DAPI. During diplotene and diakinesis, chromosomes further condensed and individualized, forming six bivalents (Figure 7A and 7B) [3]. In $m r g-1$ (qa6200) and mrg-1(tm 1227) mutant germ lines, the mitotic nuclei entered the transition zone as in the wild type; however, leptotene/zygotene-nuclei with polarized crescent-like morphology were distributed in the germline regions corresponding to the mid to late pachytene regions in the wild-type gonad, indicative of a failure of the polarized chromatin to redisperse and align longitudinally (Figure 7C and 7E). Consistent with this, few nuclei with parallel tracks were observed in $\mathrm{mrg}$ 1(lf) germ lines (Figure 7D and 7F). Similarly, in syp2(ok307) synapsis-defective mutants polarized chromosomes persisted from the transition zone through almost the whole pachytene region (Figure $7 \mathrm{G}$ and $7 \mathrm{H}$ ) [33]. In contrast, no obvious defects in nuclear progression from leptotene/zygotene to pachytene were found in brc- 1(tm1145) mutants lacking the brc-1 gene that is essential for DSB repair through inter-sister recombination but dispensable for chromosome crossover (Figure 7I and 7J) [36]. These findings indicate that the progression of germline nuclei from leptotene/zygotene to pachytene is defective in $m r g-1(l f)$ germ lines, which may result from impaired synapsis and contribute to the activation of synapsis checkpoint.

mrg-1 does not affect chromosomal loading of the SC

To evaluate the effect of $m r g-1$ on synapsis, we first used the $5 \mathrm{~S}$ rDNA located on the non-pairing center region of the fifth chromosome as a probe to examine the pairing of chromosomes by fluorescence in situ hybridization (FISH) [9]. Our results indicated that in $\mathrm{mrg}_{-}$ 1(qa6200) mutants chromosome pairing was reduced in gonadal regions corresponding to premeiotic to early pachytene zones (Figure $8 \mathrm{~A}$ and $8 \mathrm{~B}$, gonad regions 2, 3 and 4) compared to the wild-type germ line, but no obvious defect was observed in the more proximal germline regions (Figure 8A and 8B), suggesting that chromo- 
some pairing is slightly perturbed in the absence of $\mathrm{mrg}$ 1. Next, we performed immunostaining of REC-8 to examine whether $m r g-1$ mutants exhibited defects in sister chromatid cohesion. In wild type, REC-8 distributed along the axes of sister chromatids in pachytene stage nuclei and it persisted on condensed chromosomes at diakinesis (Figure 8C). Similar REC-8 staining was observed in the pachytene region in $\mathrm{mrg}-1$ (qa6200) mutants. In addition, REC- 8 was also found on each chromosomal piece in $\mathrm{mrg}-1$ (qa6200) oocytes containing 6 or $>6$ DAPI-stained bodies (Figure 8C). These results sug- gest that loss of mrg- 1 function does not cause obvious defect in sister chromatid cohesion. Finally, we asked whether the chromosomal loading of the SC was affected in $\mathrm{mrg}-1$ mutants by examining the co-existence of HTP3 and SYP-2, a lateral element and a central element of the SC, respectively. We found that HTP-3 and SYP-2 colocalized well along the axes of chromosomes in $\mathrm{mrg}$ 1 (qa6200) mutants, as observed in wild-type worms (Figure 8D), suggesting that loss of $m r g-1$ does not obviously affect chromosomal loading of the SC.
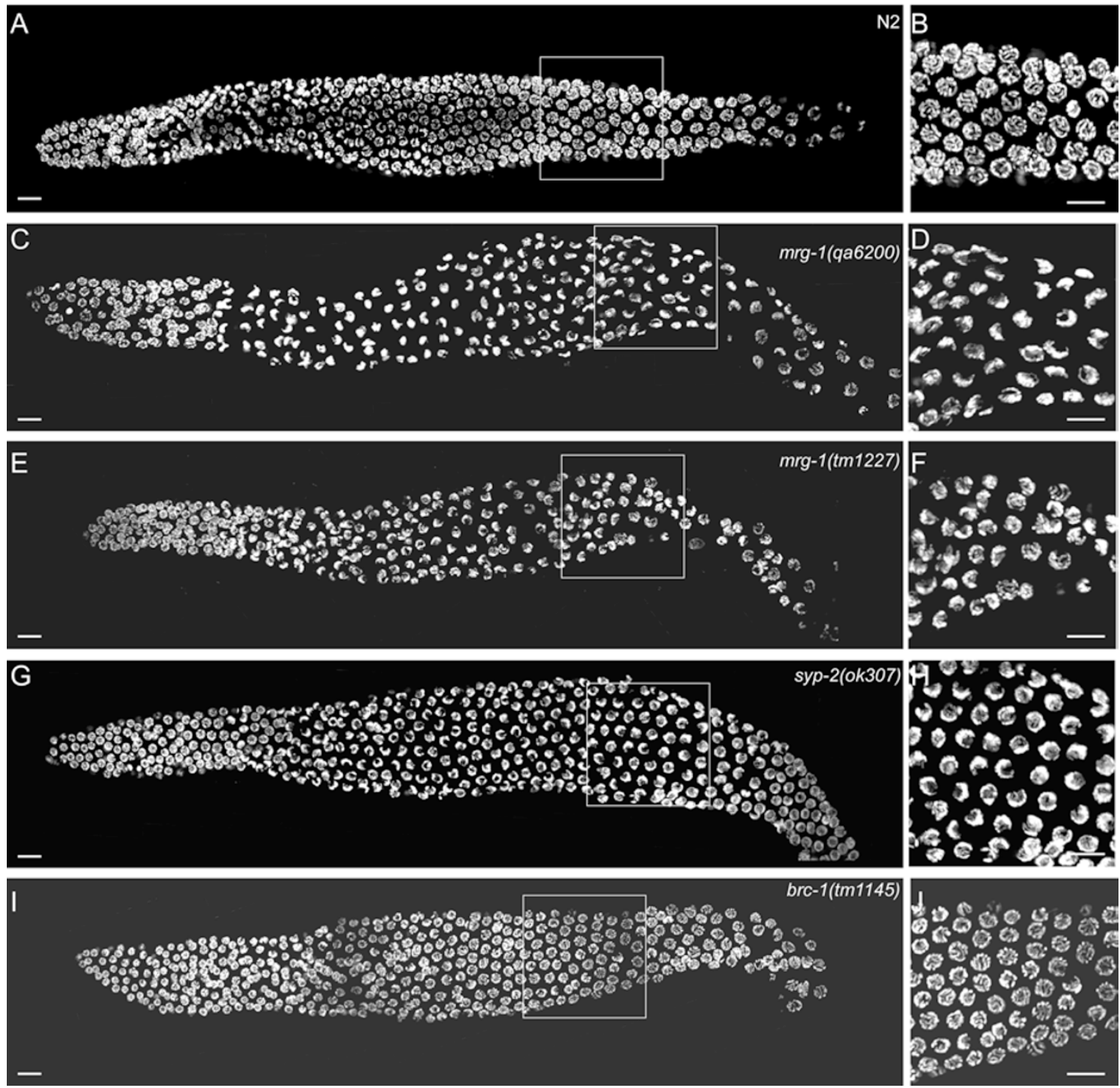

Figure 7 mrg-1 mutants are defective in progression of meiotic prophase. Representative images of DAPI-stained nuclei of whole-mount gonads from age-matched worms of the following strains are shown: (A, B) N2, (C, D) mrg-1(qa6200), (E, F) mrg-1(tm1227), (G, H) syp-2(ok307), and (I, J) brc-1(tm1145). Boxed regions in A, C, E, G and I representing mid to late pachytene stages are magnified and shown in $\mathbf{B}, \mathbf{D}, \mathbf{F}, \mathbf{H}$ and $\mathbf{J}$, respectively. $\geq 19$ gonads for each strain were analyzed. Bars, $10 \mu \mathrm{m}$. 
A

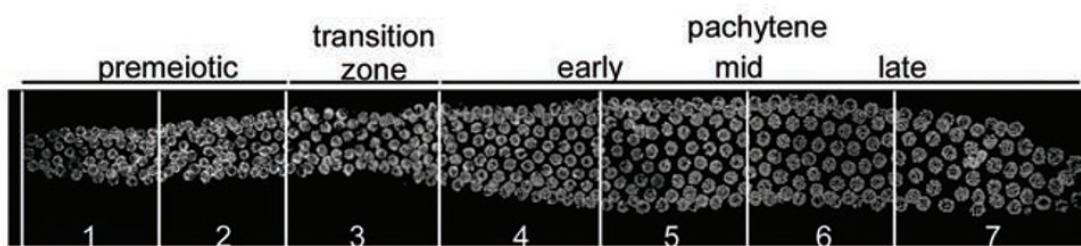

B
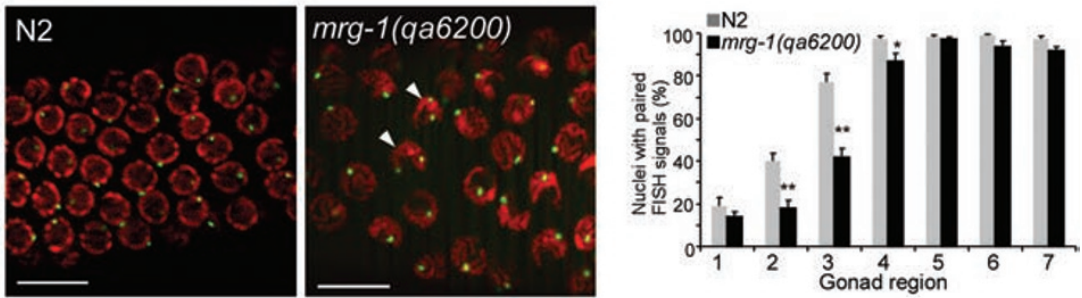

C
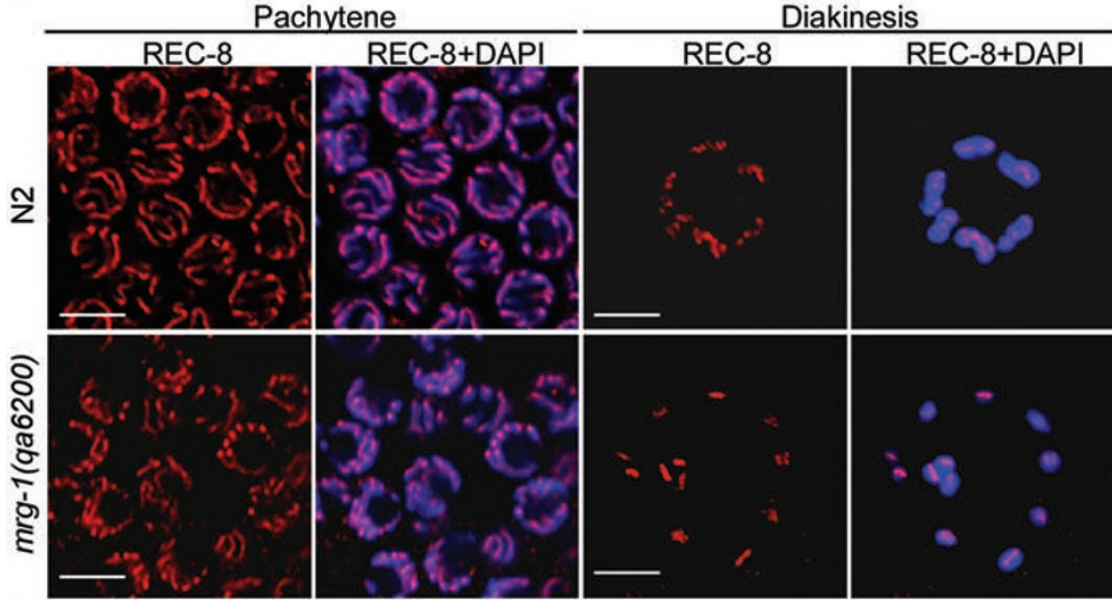

Diakinesis

D

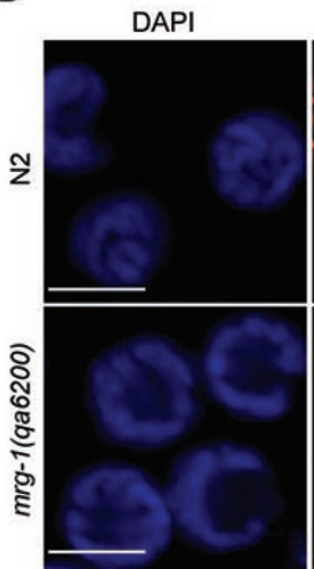

HTP-3
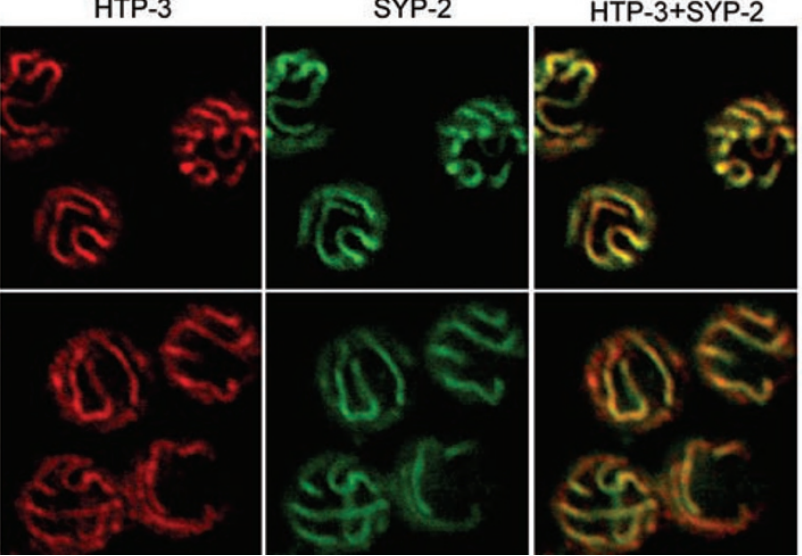

Figure 8 Analysis of chromosome pairing, sister chromatid cohesion, and chromosomal loading of the SC in $m r g-1$ mutants. (A) Representative image of a wild-type germ line with regions of meiotic progression indicated. (B) Representative FISH images of mid pachytene nuclei in N2 (left) and mrg-1(qa6200) (middle) germ lines using 5S rDNA (green) on chromosome V. DAPI staining is shown in red. Arrowheads indicate nuclei with unpaired FISH signals. Bars, $10 \mu \mathrm{m}$. Quantification of nuclei with paired FISH signals in different germline regions is indicated in the right panel. For each strain, 7 gonads were examined and a total of $\geq 74$ nuclei were analyzed for each zone. Comparisons were performed between N2 and mrg-1(qa6200) mutants using unpaired $t$-test. ${ }^{*} P<0.05,{ }^{* *} P<0.001$. (C) Representative images of REC-8 antibody staining (red) in pachytene and diakinesis nuclei in N2 and mrg-1(qa6200) worms. DAPI staining is shown in blue. Bars, $5 \mu \mathrm{m}$. (D) Representative images of HTP-3 (red) and SYP-2 (green) antibody staining in mid pachytene nuclei in N2 and mrg-1(qa6200) germ lines. DAPI staining is indicated in blue. Bars, $5 \mu \mathrm{m}$. 
mrg-1 mutants have increased germline $\mathrm{H} 3 \mathrm{~K} 9 \mathrm{me} 2$, similar to mutants defective in synapsis

Because MRG-1 is homologous to yeast Eaf3/Alp13 and mammalian MRG15, which are subunits of the NuA4/Tip60 acetyltransferase complex, we sought to determine whether the acetylation of Histone $\mathrm{H} 4$ is affected in $m r g-1$ mutants using antibody staining. We did not observe an obvious change in H4K5 and H4K8 acetylation (H4K5ac and H4K8ac) between wild-type and $m r g-1$ (qa6200) worms (Figure 9A). Nevertheless, it was previously reported that dimethylation of lysine 9 of Histone H3 (H3K9me2), which marks heterochromatin and transcriptionally inactive regions, is enriched on unpaired chromosomes and chromosome regions [3739]. Thus, we examined $\mathrm{H} 3 \mathrm{~K} 9 \mathrm{me} 2$ in $m r g-1$ mutants. As described previously [40], in wild-type germ lines nuclei from the early to mid pachytene displayed small foci of $\mathrm{H} 3 \mathrm{~K} 9 \mathrm{me} 2$. From the late pachytene to diplotene, H3K9me2 staining was strongly increased. The gradual increase in $\mathrm{H} 3 \mathrm{~K} 9 \mathrm{me} 2$ may reflect the transition from a looser chromatin structure in the early pachytene stage to a more condensed structure in the diplotene stage (Figure 9B). In contrast, mrg-1(qa6200) germline nuclei maintained a much higher level of $\mathrm{H} 3 \mathrm{~K} 9 \mathrm{me} 2$ from the early pachytene region to diplotene, suggesting that the chromatin adopts a more condensed structure (Figure 9B). A similar increase in $\mathrm{H} 3 \mathrm{~K} 9 \mathrm{me} 2$ level was observed in syp-2(ok307) and him-3(e1256) synapsis-defective mutants (Figure 9B and data not shown) [39]. By contrast, H3K9me2 in spo-11(ok79) mutants, in which meiotic recombination is disrupted but synapsis progression is not affected, was similar to that in wild type (Figure 9B). To assess the effect of $\mathrm{H} 3 \mathrm{~K} 9 \mathrm{me} 2$ on meiotic progression, we used RNAi to deplete met-2 [40] and ego-1 [41], which encode a methyltransferase responsible for $\mathrm{H} 3 \mathrm{~K} 9 \mathrm{me} 2$ and an RNA-dependent RNA polymerase required for H3K9me 2 enrichment on unpaired chromosome regions during meiosis, respectively. In both wild type and $\mathrm{mrg}$ 1(qa6200) mutants RNAi of met- 2 and ego-1 caused a strong decrease in $\mathrm{H} 3 \mathrm{~K} 9 \mathrm{me} 2$ level; however, these RNAi treatments did not change the chromatin morphology compared with control RNAi (Figure 9C). In addition, met-2 RNAi did not inhibit the chromosome fragmentation in $m r g-1(l f)$ mutants after exposure to $\gamma$-irradiation (Figure 4C). Taken together, these results suggest that the elevated $\mathrm{H} 3 \mathrm{~K} 9 \mathrm{me} 2$ is not responsible for the meiotic progression defects and DSB repair defects in $m r g-1$ mutants. Instead, it is likely to be a mark of chromosome regions with impaired synapsis at pachytene stage, which further suggests that MRG-1 likely plays a role in regulating chromosome synapsis.

\section{Discussion}

In an effort to search for factors whose inactivation may cause abnormal germ cell apoptosis in C. elegans, we have identified the chromodomain-containing protein MRG-1 as an important factor for maintenance of genomic integrity during meiosis. We found that loss of $m r g-1$ function led to an elevation in germ cell apoptosis. Importantly, the increase in apoptosis in $\mathrm{mrg}$-1(lf) germ lines was partially inhibited by mutations affecting either genes involved in the DNA damage signaling pathway or the synapsis checkpoint gene pch-2. These findings suggest that the elevation in germ cell apoptosis likely results from defective DSB repair and/or impaired synapsis during the prophase of meiosis I. In agreement with this hypothesis, inactivation of both DNA damage and chromosome synapsis checkpoints abrogated the elevated germ cell apoptosis. Thus the requirement of these signaling pathways in the absence of $m r g-1$ suggests that MRG-1 is important for proper DSB repair and synapsis in the prophase of meiosis I.

In support of the notion that MRG-1 is important for DSB repair, we found that $m r g-1(l f)$ worms accumulated spo-11-dependent RAD-51 foci from mid to late pachytene stages in germ lines, suggesting that DSBs are generated normally but fail to be repaired in a timely fashion in meiosis. Such DSB repair defects likely lead to the activation of DNA damage checkpoint pathway for triggering apoptosis. In addition, $m r g-1(l f)$ worms exhibited excessive apoptosis and more chromosome fragmentation following exposure to DNA-damaging agents, indicating that MRG-1 is also essential for repair of DNA lesions generated exogenously, which provides further evidence that MRG-1 plays an important role in DNA repair in $C$. elegans. On the other hand, we found that $m r g-1$ RNAi still induced obvious apoptosis in spo-11 mutants in which no DSB was generated, and $m r g-1(l f)$ caused apoptosis was partially dependent on the synapsis checkpoint gene $p c h-2$, suggesting that MRG-1 likely plays a role in synapsis and its loss of function triggers the synapsis checkpoint. To figure out how MRG-1 may function in this respect, we examined several events leading to chromosome synapsis, including sister chromatid cohesion, homologous chromosome pairing, and chromosomal loading of the SC. To our surprise, we only detected slight delay in chromosome pairing in $\mathrm{mrg}$-1(qa6200) mutants, whereas no obvious defects was found in sister chromatid cohesion and chromosomal loading of the SC. Thus the weak defect in chromosome pairing might result from impaired synapsis, but not a direct aftermath of failure in chromosome pairing. Importantly, we found that chromosomes polarized at leptotene/zygotene stages fail 
A

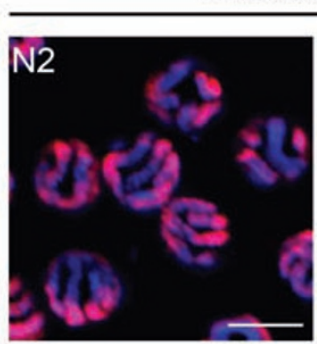

H4K5ac+DAPI

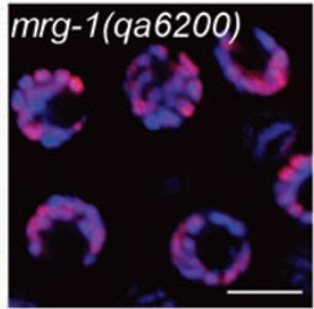

H4K8ac+DAPI

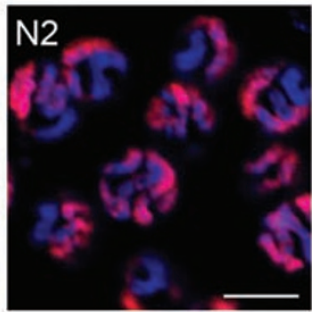

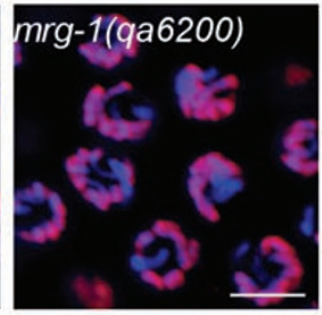

B
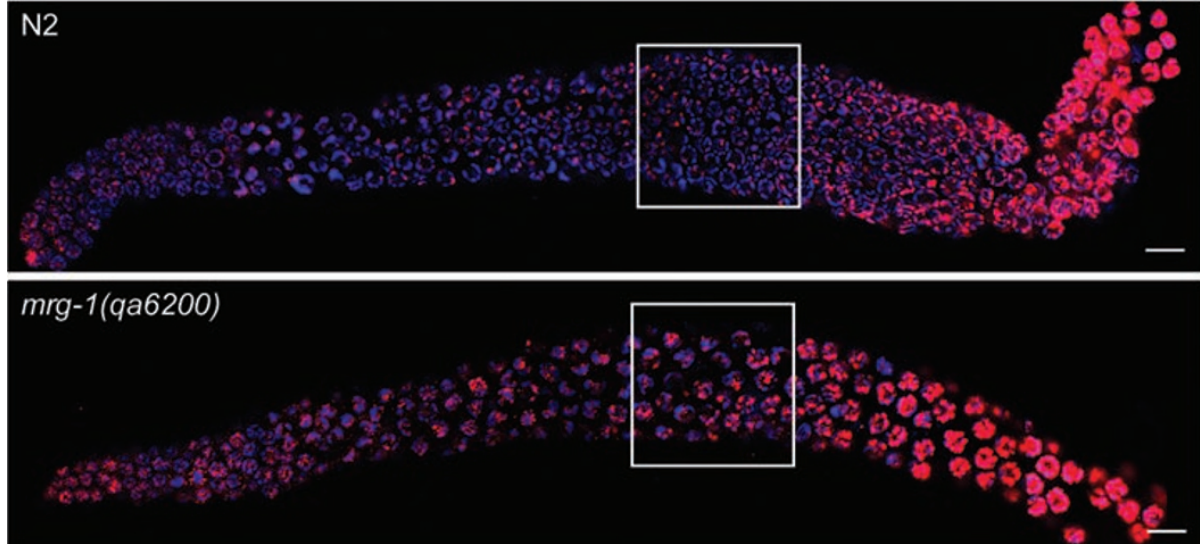

syp-2(ok307)
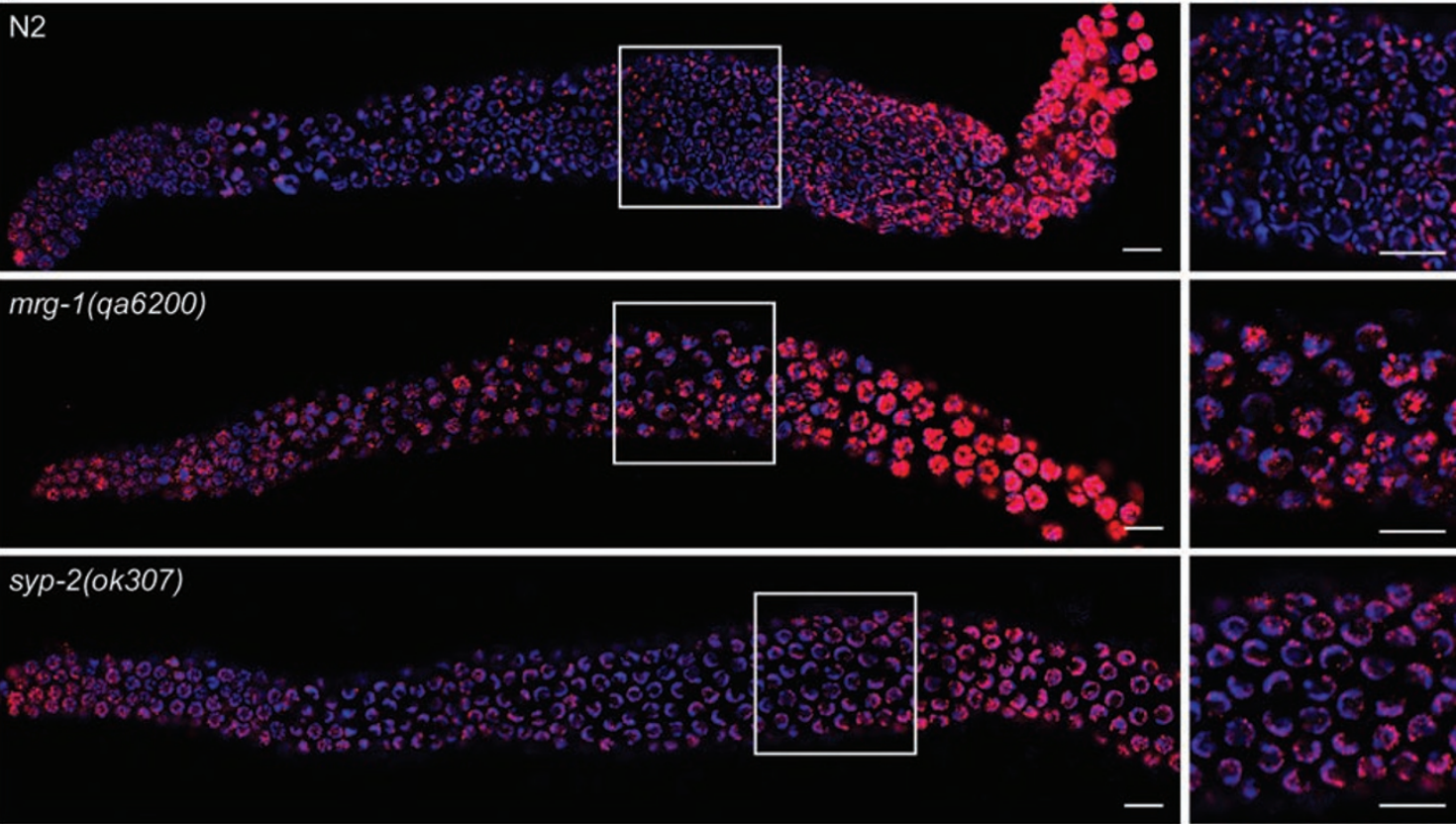

spo-11(ok79)

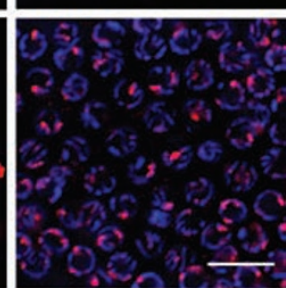

C

N2

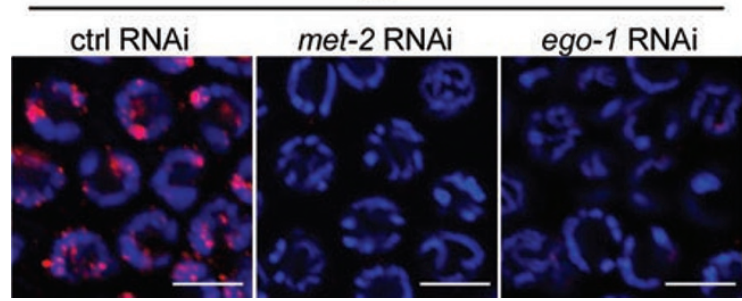

$\operatorname{mrg}-1(q a 6200)$

\begin{tabular}{|l|r|r}
\hline \multicolumn{1}{c|}{ ctrl RNAi } & met-2 RNAi & ego-1 RNAi \\
& & \\
& & \\
& & \\
\hline
\end{tabular}

Figure 9 mrg-1 and syp-2 mutants show increased H3K9me2 in the germ line. (A) Representative images of H4K5ac (left) and H4K8ac (right) of nuclei in the pachytene regions of N2 and mrg-1(qa6200) germ lines. Bars, $5 \mu \mathrm{m}$. (B) Representative images of H3K9me2 patterns in N2, mrg-1(qa6200), syp-2(ok307), and spo-11(ok79) germ lines. The boxed regions in the left panels are magnified and shown in the right panels. Bars, $10 \mu \mathrm{m}$. (C) Representative images of H3K9me2 in nuclei of late pachytene regions in N2 and mrg-1(qa6200) germ lines treated with RNAi of met-2 and ego-1. Bars, $5 \mu \mathrm{m}$. In all panels DAPI-stained chromatins are shown in blue and antibody staining is shown in red. 
to redisperse and align along their entire lengths at the pachytene stage in $m r g-1$ mutants, which is analogous to the defective progression of meiosis caused by synapsis failure in mutants deficient in the SC, suggesting that synapsis is likely not fully completed in the absence of $m r g-1$ although chromosomal loading of the SC is not obviously affected. We reason that MRG-1 may be involved in the regulation of synapsis progression through some yet unknown mechanisms.

Eaf3/Alp13 and MRG15, the MRG-1 homologs in yeast and mammals, respectively, are components of the NuA4/Tip60 HAT complex that acts at multiple levels to affect DNA damage response (DDR) and DNA repair. NuA4/Tip60 is recruited to the chromatin surrounding DSBs to facilitate DNA repair through chromatin modifications such as acetylation of the tail of Histone H4, thus opening and relaxing the chromatin for access of DNA repair proteins $[28,42]$. Tip60 can directly acetylate the ATM (ataxia-telangiectasia mutated) kinase, promoting its activation in response to DNA damage [28]. Moreover, the acetyltransferase activity of Tip60 is stimulated by binding of its chromodomain to $\mathrm{H} 3 \mathrm{~K} 9 \mathrm{me} 3$ [43]. Whether MRG-1/MRG15 affects DSB repair by regulating the HAT activity of Tip60 remains to be explored, though it was found that histone acetylation and recruitment of initial DNA repair proteins such as 53BP1 were delayed in $\mathrm{Mrg} 15$-null mouse embryonic fibroblasts following $\gamma$-irradiation [27]. In C. elegans, loss of $\mathrm{mrg}$ 1 caused accumulation of RAD-51 foci, suggesting that MRG-1 should play a role downstream of the formation of the nucleoprotein filament in the process of DSB repair. Nevertheless, we did not detect an obvious change in either H4K5ac or H4K8ac in mrg- 1 mutant germ line. Thus it remains to be further investigated whether MRG1 affects the HAT activity of the Tip60 complex on other sites. Interestingly, using the yeast two-hybrid assay, we found that MRG-1 directly interacts with the DNA helicase RUVB-2, a homolog of another component of the Tip60 complex, RVB1/2/Tip49 [44] (data not shown). RVB1/2/Tip49 is important for the HAT activity of the Tip60 complex [42]. Notably, the prokaryotic homologs of RVB1/2, RuvABC, promote branch migration and Holliday junction processing [45]. Thus the interaction between MRG-1 and RUVB-2 may promote the recruitment of DNA repair proteins other than RAD-51 to DNA breaks, and/or facilitate the processing of Holliday junctions in HR-mediated DSB repair.

Recently, it was found that loss of spr-5, which encodes a histone H3K4me2 demethylase, led to elevated levels in germline $\mathrm{H} 3 \mathrm{~K} 4 \mathrm{me} 2$, p53-dependent apoptosis, and meiotic RAD-51 foci, suggesting that histone modifications play a role in meiotic DSB repair in C. elegans
[46]. Intriguingly, we found that dimethylation of the lysine 9 of histone H3 was similarly increased from the early to late pachytene regions in mutants deficient in $m r g-1$, syp-2, and him-3, compared with wild-type germ lines. However, RNAi depletion of met-2 or ego-1, which are essential for generation of $\mathrm{H} 3 \mathrm{~K} 9 \mathrm{me} 2$ and enrichment of $\mathrm{H} 3 \mathrm{~K} 9 \mathrm{me} 2$ on unpaired chromosome regions, respectively [40, 41], failed to restore the progression of synapsis in $\mathrm{mrg}-1$ (qa6200) mutants. Thus the increase in H3K9me2 is likely to be a mark of chromosome regions with impaired synapsis instead of playing a causative role. In addition, whether the elevation in $\mathrm{H} 3 \mathrm{~K} 9 \mathrm{me} 2$ has a direct effect on meiotic DSB repair needs to be further explored. As MRG-1 directly interacts with RUVB-2 and ZK1127.3 [47], the homologs of the NuA4/Tip60 subunits RVB1 and Eaf7/MRGBP, respectively, it remains possible that these interactions may affect histone acetylation and/or methylation at certain sites during chromosome synapsis and/or DSB repair. Alternatively, MRG1 may affect the progression of meiotic prophase by regulating the expression of genes involved in synapsis and DSB repair since MRG-1 was found to be required for germline gene silencing [18]. Further elucidation of how MRG-1 functions will provide deep insights into the molecular mechanisms underlying DSB repair, synapsis, and maintenance of genomic integrity.

\section{Materials and Methods}

\section{C. elegans strains and genetics}

C. elegans strains were cultured at $20^{\circ} \mathrm{C}$ and maintained with standard procedures [48]. The Bristol N2 strain was used as wild type. Mutant and balancer strains used are listed by linkage groups:

LG I: cep-1(gk138), hus-1(op244); hT2[bli-4(e937) let-?(q782) qIs 48] (I;III).

LG II: $m r t-2(e 2663), p c h-2(t m 1458), v p s-18(t m 1125)$.

LG III: brc-1(tm1145), clk-2(mn159), mrg-1(ok1262), mrg1(qa6200), mrg-1(tm 1227), qC1 [dpy-19(e1259)glp-1(q339) qIs 26(lag-2::GFP)](III; V).

LG IV: ced-3(n717), him-3(e1256), spo-11(ok79), nT1[unc(n754) let qIs50](IV;V).

LG V: syp-2(ok307), egl-1(n1084n3082).

Deletion strains were outcrossed with the N2 strain at least four times. Double mutants were constructed with standard protocols [49]. smIs34 is an integrated array expressing CED-1::GFP [24]. $\mathrm{M}+\mathrm{Z}$ - mrg-1 hermaphrodites (homozygous hermaphrodites from heterozygous mothers; M, maternal load; Z, zygotic synthesis) were used for all germline apoptosis and meiosis analyses.

\section{RNA interference}

RNAi clones of mrg-1, met-2, and ego-1 were obtained from the C. elegans RNAi library generated by the Ahringer laboratory and verified by sequencing. RNAi was performed at $20{ }^{\circ} \mathrm{C}$ using a feeding assay. Briefly, worms synchronized at early larval stage 
4 (L4) with different genetic backgrounds were grown on plates seeded with the bacterial strain HT115 (DE3) expressing dsRNAs of $m r g-1$, met-2 and ego-1. The progeny were maintained on RNAi plates and worms with intact germ lines after reaching adult age were synchronized for germ cell corpse quantification and other experimental analyses (see below). The efficiency of $m r g-1$ RNAi was verified by examining the loss of germ lines in certain proportion of adult worms.

\section{Germ cell apoptosis analysis and DNA damage treatment}

Germ cell corpses were viewed by formation of disc-like structures under DIC optics. Alternatively, cell corpses were recognized by formation of CED-1::GFP rings in worms carrying the integrated array smIs 34 . For time course analysis of apoptosis, synchronized worms at $24,36,48$, and $60 \mathrm{~h}$ post L 4 stage were scored for germ cell corpses under DIC optics. Cell corpses of one gonad arm were scored for each worm and a total of $\geq 15$ worms of each strain were examined at every time point. All experiments were repeated 2-3 times and the average number of germ cell corpses per gonad arm and the SEM were calculated from worms ( $\geq 30$ totally) examined for each strain at every time point. For DNA damage treatment, worms at $12 \mathrm{~h}$ post L4 stage were irradiated with different dosages of $\gamma$-rays $(30,60$, and $120 \mathrm{~Gy})$ or treated with ENU at different concentrations $(0,2.5$, and $5 \mathrm{mM})$ for $4 \mathrm{~h}$. Worms were then recovered for $24 \mathrm{~h}$ and scored for germ cell corpses under DIC optics as described above. The experiments were repeated twice. Data comparisons were performed using the unpaired $t$-test.

To examine $\gamma$-irradiation-induced chromosome fragmentation, synchronized L4 worms were irradiated with 70 and $90 \mathrm{~Gy}$, respectively, and back to culture for another $48 \mathrm{~h}$. Gonads were then dissected out and diakinesis nuclei were stained with DAPI and observed under a fluorescence scope (Zeiss Axioimager M1).

\section{Analysis of meiotic defects in mrg-1(lf) worms}

The number of chromosomes in oocytes was examined by DAPI staining. Briefly, gonads of N2 and $\mathrm{M}+\mathrm{Z}-\mathrm{mrg}-1$ hermaphrodites at the age of $48 \mathrm{~h}$ post L4 stage were dissected in phosphate buffer containing $0.25 \mathrm{mM}$ levamisole and fixed with $2 \%$ formaldehyde. After extensive wash, gonads were stained with DAPI (2 $\mu \mathrm{g} / \mathrm{ml}$ ) and viewed under fluorescence scope. To quantify brood sizes and proportions of males, $\mathrm{M}+\mathrm{Z}-\mathrm{mrg}-1$ mutants or $\mathrm{mrg}$ 1(RNAi) worms at L4 stage with intact germ lines were chosen to lay eggs on NGM plates. The total numbers of M-Z- $m r g-1$ eggs laid and hatched larvae were scored.

\section{Antibodies and immunostaining}

RAD-51 polyclonal antibody was generated in rabbits by injecting a GST-fused N-terminal peptide (amino acids 1-103) of RAD51. SYP-2 polyclonal antibody was raised in mice by injecting a GST-fused C-terminal peptide (amino acids 101-213) of SYP-2. The RAD-51 and SYP-2 sera were affinity purified and used with 1:500 and 1:200 dilution, respectively. HTP-3 polyclonal antibody was generated in guinea pig by injecting His6-tagged HTP-3 (amino acids 414-607) protein and used with a dilution of 1:200. Rabbit polyclonal REC-8 antibody was purchased from Novus Biologicals (\#29470002) and used with 1:500 diluton. Rabbit polyclonal antibodies against acetylated $\mathrm{H} 4 \mathrm{~K} 5$ and $\mathrm{H} 4 \mathrm{~K} 8$ were purchased from Upstate Biotechnology Inc. and used with 1:1 000 dilution. Mouse monoclonal antibody for $\mathrm{H} 3 \mathrm{~K} 9 \mathrm{me} 2$ was purchased from
Abcam (\#ab1220) and used with 1:500 dilution. For immunostaining, worm gonads were dissected out from hermaphrodites at 21$24 \mathrm{~h}$ post L 4 stage and fixed in $4 \%$ paraformaldehyde for $40 \mathrm{~min}$ at room temperature. Samples were then sequentially treated with methanol, methanol:acetone (1:1), and acetone for 5 min each at $-20{ }^{\circ} \mathrm{C}$. After being washed three times in phosphate buffer (1.1 $\mathrm{mM} \mathrm{KH} \mathrm{PO}_{4}, 153.8 \mathrm{mM} \mathrm{NaCl}, 3.0 \mathrm{mM} \mathrm{Na} \mathrm{HPO}_{4}, \mathrm{pH}$ 7.4) containing $1 \%$ Triton $\mathrm{X}-100$, samples were sequentially incubated with primary antibodies for $16-18 \mathrm{~h}$ at $4{ }^{\circ} \mathrm{C}$ and FITC- or Cy3conjugated secondary antibodies (1:200 and 1:4 000 dilution, respectively) for $2 \mathrm{~h}$ at room temperature. An antifade solution (Vector Laboratories) containing $2 \mu \mathrm{g} / \mathrm{ml}$ DAPI was added to samples before imaging under a confocal microscope (Olympus Fluoview FV1000, IX81 inverted laser scanning microscope).

\section{Fluorescence in situ hybridization}

$5 \mathrm{~S}$ rDNA [9] was amplified by polymerase chain reaction (PCR) and labeled with digoxigenin-16-dUTP (Roche) by nick translation. Worm gonads fixed on slides were denatured at $80{ }^{\circ} \mathrm{C}$ for $2 \mathrm{~min}$ in $2 \times \mathrm{SSC}\left(0.3 \mathrm{mM} \mathrm{NaCl}, 0.03 \mathrm{mM} \mathrm{Na}_{3} \mathrm{C}_{6} \mathrm{H}_{5} \mathrm{O}_{7}, \mathrm{pH} 7.0\right)$ containing $70 \%$ formamide and then dehydrated by sequentially immersing in a pre-cooled ethanol series $(70 \%, 90 \%$, and $100 \%$ for $5 \mathrm{~min}$ each). 50-100 ng probe labeled with digoxigenin was denatured and then incubated with worm gonads on slides at $37^{\circ} \mathrm{C}$ overnight in a moist chamber. Slides were subsequently washed once in $2 \times \mathrm{SSC}$ for $10 \mathrm{~min}$ at $42{ }^{\circ} \mathrm{C}$ and twice in $2 \times \mathrm{SSC}$ for $5 \mathrm{~min}$ each at room temperature. Chromosomes were counterstained with DAPI in an antifade solution (Vector Laboratories).

\section{Acknowledgments}

We thank the C. elegans Genetic Center (CGC) and Dr Shohei Mitani (Tokyo Women's Medical Univeristy, Japan) for providing worm deletion strains; Dr Xiaochen Wang (National Institute of Biological Sciences, China) for helpful suggestions and Dr Isabel Hanson for proofreading of the manuscript. This research was supported by grants from the National Basic Research Program of China (2011CB910102 and 2007CB947201) and the National Natural Science Foundation of China (31025015 and 30871266). CY is supported by the Hundred Talents Program of the Chinese Academy of Sciences.

\section{References}

1 Sancar A, Lindsey-Boltz LA, Unsal-Kacmaz K, Linn S. Molecular mechanisms of mammalian DNA repair and the DNA damage checkpoints. Annu Rev Biochem 2004; 73:39-85.

2 Hanahan D, Weinberg RA. The hallmarks of cancer. Cell 2000; 100:57-70.

3 Garcia-Muse T, Boulton SJ. Meiotic recombination in Caenorhabditis elegans. Chromosome Res 2007; 15:607-621.

4 Lemmens BB, Tijsterman M. DNA double-strand break repair in Caenorhabditis elegans. Chromosoma 2011; 120:1-21.

5 Colaiacovo MP. The many facets of SC function during $C$. elegans meiosis. Chromosoma 2006; 115:195-211.

6 Severson AF, Ling L, van Zuylen V, Meyer BJ. The axial element protein HTP-3 promotes cohesin loading and meiotic axis assembly in C. elegans to implement the meiotic program of chromosome segregation. Genes Dev 2009; 23:1763-1778. 
7 Smolikov S, Schild-Prufert K, Colaiacovo MP. A yeast twohybrid screen for SYP-3 interactors identifies SYP-4, a component required for synaptonemal complex assembly and chiasma formation in Caenorhabditis elegans meiosis. PLoS Genet 2009; 5:e1000669.

8 Smolikov S, Eizinger A, Schild-Prufert K, et al. SYP-3 restricts synaptonemal complex assembly to bridge paired chromosome axes during meiosis in Caenorhabditis elegans. Genetics 2007; 176:2015-2025.

9 Dernburg AF, McDonald K, Moulder G, Barstead R, Dresser M, Villeneuve AM. Meiotic recombination in C. elegans initiates by a conserved mechanism and is dispensable for homologous chromosome synapsis. Cell 1998; 94:387-398.

10 Rinaldo C, Bazzicalupo P, Ederle S, Hilliard M, La Volpe A. Roles for Caenorhabditis elegans rad-51 in meiosis and in resistance to ionizing radiation during development. Genetics 2002; 160:471-479.

11 Couteau F, Zetka M. HTP-1 coordinates synaptonemal complex assembly with homolog alignment during meiosis in $C$. elegans. Genes Dev 2005; 19:2744-2756.

12 Martinez-Perez E, Villeneuve AM. HTP-1-dependent constraints coordinate homolog pairing and synapsis and promote chiasma formation during C. elegans meiosis. Genes Dev 2005; 19:2727-2743.

13 Bhalla N, Dernburg AF. A conserved checkpoint monitors meiotic chromosome synapsis in Caenorhabditis elegans. Science 2005; 310:1683-1686.

14 Gartner A, Boag PR, Blackwell TK. Germline survival and apoptosis. WormBook 2008; 1-20.

15 Hofmann ER, Milstein S, Boulton SJ, et al. Caenorhabditis elegans HUS-1 is a DNA damage checkpoint protein required for genome stability and EGL-1-mediated apoptosis. Curr Biol 2002; 12:1908-1918.

16 Yang M, Sun J, Sun X, Shen Q, Gao Z, Yang C. Caenorhabditis elegans protein arginine methyltransferase PRMT-5 negatively regulates DNA damage-induced apoptosis. PLoS Genet 2009; 5:e1000514.

17 Schumacher B, Hanazawa M, Lee MH, et al. Translational repression of $C$. elegans 53 by GLD-1 regulates DNA damageinduced apoptosis. Cell 2005; 120:357-368.

18 Takasaki T, Liu Z, Habara Y, et al. MRG-1, an autosomeassociated protein, silences X-linked genes and protects germline immortality in Caenorhabditis elegans. Development 2007; 134:757-767.

19 Fujita M, Takasaki T, Nakajima N, Kawano T, Shimura Y, Sakamoto H. MRG-1, a mortality factor-related chromodomain protein, is required maternally for primordial germ cells to initiate mitotic proliferation in C. elegans. Mech Dev 2002; 114:61-69.

20 Pena AN, Pereira-Smith OM. The role of the MORF/MRG family of genes in cell growth, differentiation, DNA repair, and thereby aging. Ann N Y Acad Sci 2007; 1100:299-305.

21 Tominaga K, Kirtane B, Jackson JG, et al. MRG15 regulates embryonic development and cell proliferation. Mol Cell Biol 2005; 25:2924-2937.

22 Bender LB, Suh J, Carroll CR, et al. MES-4: an autosomeassociated histone methyltransferase that participates in silencing the $\mathrm{X}$ chromosomes in the $C$. elegans germ line. Development 2006; 133:3907-3917.
23 Xiao H, Chen D, Fang Z, et al. Lysosome biogenesis mediated by vps-18 affects apoptotic cell degradation in Caenorhabditis elegans. Mol Biol Cell 2009; 20:21-32.

24 Chen D, Xiao H, Zhang K, et al. Retromer is required for apoptotic cell clearance by phagocytic receptor recycling. Science 2010; 327:1261-1264.

25 Bertram MJ, Berube NG, Hang-Swanson X, et al. Identification of a gene that reverses the immortal phenotype of a subset of cells and is a member of a novel family of transcription factor-like genes. Mol Cell Biol 1999; 19:1479-1485.

26 Kusch T, Florens L, Macdonald WH, et al. Acetylation by Tip60 is required for selective histone variant exchange at DNA lesions. Science 2004; 306:2084-2087.

27 Garcia SN, Kirtane BM, Podlutsky AJ, Pereira-Smith OM, Tominaga K. Mrg15 null and heterozygous mouse embryonic fibroblasts exhibit DNA-repair defects post exposure to gamma ionizing radiation. FEBS Lett 2007; 581:5275-5281.

28 Squatrito M, Gorrini C, Amati B. Tip60 in DNA damage response and growth control: many tricks in one HAT. Trends Cell Biol 2006; 16:433-442.

29 Stergiou L, Hengartner MO. Death and more: DNA damage response pathways in the nematode $C$. elegans. Cell Death Differ 2004; 11:21-28.

30 Schumacher B, Hofmann K, Boulton S, Gartner A. The $C$. elegans homolog of the p53 tumor suppressor is required for DNA damage-induced apoptosis. Curr Biol 2001; 11:17221727.

31 Gartner A, Milstein S, Ahmed S, Hodgkin J, Hengartner MO. A conserved checkpoint pathway mediates DNA damage-induced apoptosis and cell cycle arrest in C. elegans. Mol Cell 2000; 5:435-443.

32 Derry WB, Putzke AP, Rothman JH. Caenorhabditis elegans p53: role in apoptosis, meiosis, and stress resistance. Science 2001; 294:591-595.

33 Colaiacovo MP, MacQueen AJ, Martinez-Perez E, et al. Synaptonemal complex assembly in $C$. elegans is dispensable for loading strand-exchange proteins but critical for proper completion of recombination. Dev Cell 2003; 5:463-474.

34 Alpi A, Pasierbek P, Gartner A, Loidl J. Genetic and cytological characterization of the recombination protein RAD-51 in Caenorhabditis elegans. Chromosoma 2003; 112:6-16.

35 Bailly AP, Freeman A, Hall J, et al. The Caenorhabditis elegans homolog of Gen1/Yen1 resolvases links DNA damage signaling to DNA double-strand break repair. PLoS Genet 2010; 6:e1001025.

36 Adamo A, Montemauri P, Silva N, Ward JD, Boulton SJ, La Volpe A. BRC-1 acts in the inter-sister pathway of meiotic double-strand break repair. EMBO Rep 2008; 9:287-292.

37 Schaner CE, Kelly WG. Germline chromatin. WormBook 2006; 1-14.

38 Kelly WG, Schaner CE, Dernburg AF, et al. X-chromosome silencing in the germline of C. elegans. Development 2002; 129:479-492.

39 Bean CJ, Schaner CE, Kelly WG. Meiotic pairing and imprinted X chromatin assembly in Caenorhabditis elegans. Nat Genet 2004; 36:100-105.

40 Bessler JB, Andersen EC, Villeneuve AM. Differential localization and independent acquisition of the $\mathrm{H} 3 \mathrm{~K} 9 \mathrm{me} 2$ and H3K9me3 chromatin modifications in the Caenorhabditis 
elegans adult germ line. PLoS Genet 2010; 6:e1000830.

41 Maine EM, Hauth J, Ratliff T, Vought VE, She X, Kelly WG. EGO-1, a putative RNA-dependent RNA polymerase, is required for heterochromatin assembly on unpaired dna during C. elegans meiosis. Curr Biol 2005; 15:1972-1978.

42 Murr R, Loizou JI, Yang YG, et al. Histone acetylation by Trrap-Tip60 modulates loading of repair proteins and repair of DNA double-strand breaks. Nat Cell Biol 2006; 8:91-99.

43 Sun Y, Jiang X, Xu Y, et al. Histone H3 methylation links DNA damage detection to activation of the tumour suppressor Tip60. Nat Cell Biol 2009; 11:1376-1382.

44 Jha S, Dutta A. RVB1/RVB2: running rings around molecular biology. Mol Cell 2009; 34:521-533.

45 West SC. Processing of recombination intermediates by the RuvABC proteins. Annu Rev Genet 1997; 31:213-244.

46 Nottke AC, Beese-Sims SE, Pantalena LF, Reinke V, Shi Y, Colaiacovo MP. SPR-5 is a histone H3K4 demethylase with a role in meiotic double-strand break repair. Proc Natl Acad Sci USA 2011; 108:12805-12810.

47 Li S, Armstrong CM, Bertin N, et al. A map of the interactome network of the metazoan C. elegans. Science 2004; 303:540-543.

48 Brenner S. The genetics of Caenorhabditis elegans. Genetics 1974; 77:71-94. 